\title{
Outage Analysis and Optimization in Single- and Multiuser Wireless Energy Harvesting Networks
}

\author{
Bo Zhang, Chen Dong, Mohammed El-Hajjar, and Lajos Hanzo
}

\begin{abstract}
4 Abstract-Compared to battery-powered wireless nodes having 5 a constant but limited power supply, wireless nodes having energy 6 harvesting (EH) capability may greatly prolong the network's 7 sustainability. However, the energy usage policies (EUPs) have 8 to be carefully designed according to the characteristics of the 9 random power supply gleaned from the environment. In this 10 paper, we carry out the outage analysis of a point-to-point (P2P) 11 network relying on an EH transmitter, which has a finite energy 12 buffer (EB) for transmission over a fading channel when having 13 random energy arrival rates. A discrete Markov chain (DMC) 14 model is proposed for characterizing the energy state of the EB, 15 which is then used for quantifying the outage probability (OP) 16 over the fading channels. Then, we propose both a novel 2-D and a 17 low-complexity 1-D search algorithm for finding the specific EUPs, 18 which are capable of minimizing the OP for the P2P network 19 considered. It is shown that the EUP found by both algorithms 20 outperforms the state-of-the-art EUPs disseminated in the open 21 literature. Furthermore, we consider a multiple-access network 22 having $\boldsymbol{M} \mathbf{E H}$-aided sources, where we propose a distributed 23 EUP optimization (DEUPO) algorithm and then minimize the OP 24 relying on the local optimization of each EH-aided source.
\end{abstract}

26 Index Terms-Energy harvesting (EH), Markov chain, outage analysis, outage minimization.

\section{INTRODUCTION}

28 T $\mathrm{N}$ practical scenarios such as wireless sensor networks 30 (WSNs), it is challenging to replace the nodes; hence, 31 the network's operation is energy constrained, which is of32 ten formulated as having a limited lifetime [1]. One way of 33 circumventing this problem is allowing the nodes to harvest 34 energy from the environment. If a harvested energy source is 35 permanently available, the transceiver can be powered perpetu36 ally, which fundamentally changes the wireless system design 37 compared to the classic energy-constrained design relying on an 38 energy source storing a limited amount of energy in batteries. 39 Furthermore, based on the periodicity and magnitude of the

Manuscript received December 15, 2014; revised February 19, 2015; accepted February 27, 2015. This work was supported by the Research Councils UK (RC-UK) under the auspices of the India-UK Advanced Technology Center (IU-ATC), by the European Union's Concerto Project, by the European Research Council's Advanced Fellow Grant, and by the Royal Society's Wolfson Research Merit Award. The review of this paper was coordinated by Dr. N.-D. Đào.

B. Zhang is with the School of Electronics and Electrical Engineering, National University of Defense Technology, Changsha 410073, China (e-mail: Bo.Zhang.soton@outlook.com).

C. Dong, M. El-Hajjar, and L. Hanzo are with the School of Electronics and Computer Science, University of Southampton, Southampton SO17 1BJ, U.K. (e-mail: cd2g09@ecs.soton.ac.uk; meh@ecs.soton.ac.uk; lh@ecs.soton.ac.uk).

Color versions of one or more of the figures in this paper are available online at http://ieeexplore.ieee.org.

Digital Object Identifier 10.1109/TVT.2015.2409781 harvested energy, the transceiver may adjust its energy usage 40 policy (EUP) to improve certain network performance metrics, 41 such as the throughput or outage probability (OP). The EUP 42 may be defined as the "The policy determining the transmitting 43 power and the transmission rate, given the availability of the 44 knowledge on the amount of energy in the energy buffer, the 45 channel statistic information (CSI) as well as the noncausal 46 energy harvesting information (EHI) characterizing the energy 47 arrival rate at the transmitter."

In this paper, we investigate both the effects of random 49 energy arrival and of the EUP design on the OP of wireless 50 energy harvesting (EH) networks. Recently, the EUP design of 51 EH networks has become a hot research area. Various schemes 52 have been proposed in the literature [2]-[9] to improve certain 53 performance metrics in a particular network topology, relying 54 on different assumptions of the energy arrival rates, as well 55 as on the knowledge available at the wireless transceivers for 56 optimization.

Under the idealized simplifying assumption of having both 58 noncausal channel-state information (CSI) about the CSI to be 59 encountered in the future and about the EH information (EHI) 60 characterizing the energy arrival rate at the transmitter, in [2] 61 and [3], ${ }^{1}$ the optimal offline EUPs were designed for point-to- 62 point (P2P) networks using either the throughput maximiza- 63 tion or the file-transfer completion-time minimization as the 64 optimization objective function (OF). Later on, the authors in 65 [10] proposed the recursive geometric waterfilling algorithm for 66 solving the same problem, where more efficient recursive com- 67 putations were used for finding the optimal solutions. In [4], the 68 authors modeled both the uncertainty of the energy arrival rate 69 and that of the data arrival rate, where the transmission rate to be 70 used was determined by minimizing the average data-buffering 71 delay as the OF.

When the instantaneous CSI is not available at the transmit- 73 ter, having an outage is unavoidable for fixed-rate applications, 74 and the resultant OP of a P2P-EH network was investigated in 75 [5]-[9]. The OP analysis and OP optimization techniques may 76 be categorized into two subclasses according to the knowledge 77 of both the energy arrival rates and the mathematical framework 78 that they adopt; specifically, the first category of contributions 79 recommends the employment of time-variant policies [5], [8], 80 [9]. These authors followed the mathematical framework in [2] 81

\footnotetext{
${ }^{1}$ In [2] and [3], the terminology of "transmission policy" was used to represent the policy of using the harvested energy in the energy buffer (EB). However, the transmission policy terminology may be interpreted more widely, such as rate adaptation, multiple-access policy, etc. Therefore, to avoid ambiguity, we use the terminology of "EUP" throughout the paper.
} 
82 and [11], which adopted the directional waterfilling algorithms 83 under EH-causality constraints ${ }^{2}$ for offline EUP design com84 plemented by the stochastic dynamic programming in online 85 EUP design. The time-variant policy implies the fact that the 86 energy usage would be adapted by relying on the idealized 87 simplifying assumptions of having the a priori knowledge of 88 the instantaneous energy arrival rates. The second category of 89 EUPs recommends time-invariant policies for the long trans90 mission durations routinely encountered in WSNs, which ex91 hibit low computational complexities [6], [7]. The terminology 92 of a time-invariant policy reflects the fact that it does not rely 93 on the idealized knowledge of the instantaneous energy arrival 94 rate, regardless of whether the energy dispensation is designed 95 according to the statistical information of the energy arrival [7] 96 or not [6]. In this case, the EUP may be defined as the "The 97 policy determining the transmitting power, given the amount 98 of energy in the energy buffer and the statistical information 99 of the channel model." Against this backcloth, in this treatise, 100 we aim for filling the gap between the high-complexity time101 variant EUPS and the low-complexity state-of-the-art time102 invariant policies, by considering scenarios having a practical 103 finite EB. As we will show in this paper that the EUPs in the 104 literature [6], [7] did not exploit the EB's state and achieved 105 a suboptimal OP performance. Hence, we propose a range 106 of meritorious methods for improving the OP performance, 107 which fall into the time-invariant category to impose a low 108 computation complexity by relying merely on the knowledge 109 of the average energy arrival rate.

110 As an evolution of research in the subject area of P2P$111 \mathrm{EH}$ networks, the recent contributions on EH strategy design 112 also cover multiple-access EH networks [6], [12]-[15]. In [12], 113 Yang and Ulukus investigated the optimal packet scheduling 114 problem in the context of a two-user fading multiple-access 115 channel. In [15], Wang et al. developed optimal energy schedul116 ing algorithms for a generalized $M$-user fading multiple-access 117 channel relying on $\mathrm{EH}$, to maximize their OF constituted by 118 the network's sum rate, stipulating the idealized simplifying 119 assumption that the side information of both channel states 120 and EH states are known for a certain number of time slots 121 (TSs), where both the battery capacity and the maximum energy 122 consumption during each TS are finite. To the best of our 123 knowledge, the $O P$ minimization problem of a generalized $124 M$-user fading multiple-access channel is, however, an open 125 problem. Against this background, the novel contributions of 126 this paper are as follows.

1) An analytical framework based on a discrete Markov chain (DMC) is proposed for modeling the EB status, for the sake of investigating the OP of a P2P-EH network, in which an $\mathrm{EH}$ source node (EH-SN) equipped with a finite EB transmits to a destination node (DN). Given the EB's

\footnotetext{
${ }^{2}$ The EH-causality constraint refers to the fact that, at any time, the transceivers can only utilize the energy that was harvested during the past and the energy not harvested as yet is hence unavailable for usage. Taking into account the causality constraints imposed on the energy usage, the energy can only be saved and used in the future. Therefore, the waterfilling algorithm is redesigned as a directional one, which allows the energy flow only to take place from the past to the future.
}

size and assuming a certain probability distribution func- 132 tion (PDF) for the energy arrival rate, the OP is derived for 133 arbitrary EUPs.

2) We investigate the optimal EUP conceived for minimizing 135 the OP of a P2P-EH network. Based on our proposed ana- 136 lytical framework, we show that constructing an exhaus- 137 tive search for finding the optimal EUP for minimizing 138 the OP is impractical, owing to its excessive complexity, 139 because it scales with $\left(L_{\max }\right)$ !, where $L_{\max }$ is the number 140 of states in the DMC. Therefore, a heuristic 2-D search 141 (2D-search) algorithm is proposed for finding a meritori- 142 ous EUP; we demonstrate that the proposed algorithm is 143 potentially capable of finding the EUP at a manageable 144 complexity. $^{3}$

3) Nonetheless, the 2D-search algorithm conceived still ex- 146 hibits a high complexity; hence, we also propose a low- 147 complexity 1-D search (1D-search) algorithm. We will 148 demonstrate that the OP of the 1D-search algorithm is 149 close to that of its 2D-search counterpart, which may be 150 attractive for applications relying on low-cost hardware, 151 such as mobile phones and wireless sensors.

4) We extend the proposed DMC framework to more general 153 nonorthogonal EH networks. In contrast to the P2P sce- 154 nario, the outage events of practical EH-SNs tend to be 155 correlated. As an attractive application scenario, we will 156 investigate the OP of maximum-likelihood (ML) detection 157 in the context of spatial-division multiple-access (SDMA) 158 networks, we will decompose the OP by approximat- 159 ing it as multiple independent outage probabilities, each 160 corresponding to a simple P2P-EH-network subproblem. 161 Finally, we will propose a distributed EUP optimization 162 (DEUPO) protocol, where each EH-SN is capable of 163 optimizing its own policy using both the local statistics 164 of the fading channel and the related energy arrival model. 165

The rest of this paper is organized as follows: In Section II, 166 we first discuss the EUPs found in the literature and then invoke 167 the DMC for modeling the EB's state. Based on this model, 168 we consider the OP minimization problem and propose the 169 aforementioned 2D-search and 1D-search algorithms conceived 170 for finding the optimal EUPs. In Section III, we investigate 171 the EUP design of SDMA-EH networks, and we propose the 172 aforementioned distributed DEUPO protocol. Finally, our con- 173 clusions are presented in Section V.

II. PeEr-To-Peer-Energy Harvesting Network 175 DESIGN

\section{A. System Model and OP Formulation}

We first consider a simple P2P network constituted by an 179 $\mathrm{SN}$ and a DN, which is shown in Fig. 1. As shown in Fig. 1, 180

\footnotetext{
${ }^{3}$ When the Markov chain model has $L_{\max } \leq 10$ states and the number of OF evaluations is lower than 10 !, the exhaustive searching may be implemented and therefore may serve as the benchmark for our proposed algorithm. However, for $L_{\max }>10$, the complexity becomes excessive, which prevents us from verifying, whether the $2 \mathrm{D}$-search algorithm is capable of matching the optimal EUP. On the other hand, it is challenging to mathematically prove the optimality of a search algorithm in the context of a nonconvex problem involving highdimensional matrices. Therefore, this open problem will be further detailed in our discussions, and it will be investigated in our future work.
} 


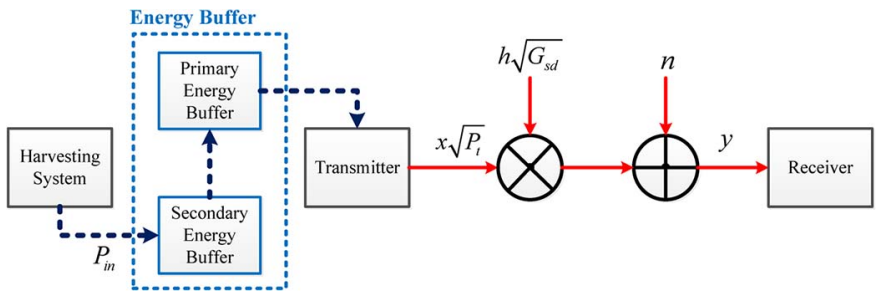

Fig. 1. System model of the P2P-EH network.

181 a primary $\mathrm{EB}$ and a secondary $\mathrm{EB}$ is required in practice 182 [1], [6]. In [1], the secondary storage is a backup storage 183 invoked for situations, when the primary storage is exhausted. 184 In [6], the authors assumed that the rechargeable energy storage 185 devices cannot charge and discharge simultaneously; hence, the 186 transmitter is powered by the primary EB for data transmission, 187 while the secondary EB is connected to the harvesting system 188 and charges up. At the end of the recharge cycle, the secondary $189 \mathrm{~EB}$ would be charged by the secondary EB. We assume that 190 the charging time of the primary EB is negligible ${ }^{4}$ and that the 191 charging efficiency is assumed to be $100 \% .^{5}$ Therefore, both 192 the primary and the secondary EBs may be represented by a 193 single EB, which is represented by the dashed-line box shown 194 in Fig. 1. This buffer is assumed to be capable of powering the 195 transmitter, while simultaneously being charged by the harvest196 ing system. We do not make any specific assumptions as to what 197 harvesting system is adopted, which may be solar cells, a wind 198 anemometer, etc., as discussed in [1]. We assume that the EB at 199 the SN has a finite EB size, where the harvested energy is stored 200 and used for transmission. We assume furthermore that the 201 energy arrival rate $P_{\text {in }}$ obeys a certain probability distribution 202 with an expectation of $\bar{P}_{\text {in }}$, and it remains constant over a TS of 203 duration $T_{E}$, while changing independently over the subsequent 204 TSs, where a time slot is a recharge cycle. We assume that 205 the instantaneous energy arrival rate is unknown and cannot be 206 used during the current TS of $T_{E}$, because the secondary EB is 207 not allowed to charge and discharge simultaneously, as shown 208 in Fig. 1. In order to focus our attention on the EUP conceived 209 for wireless transmission, we assume that the circuit power con210 sumption at the $\mathrm{SN}$ is negligible and that the energy conversion 211 efficiency between the EB and the transmit power is $100 \%{ }^{6}$

212 Let us now consider the channel modeling of the wireless 213 communication links. We consider a narrow-band block-fading 214 channel model, where the fading coefficients remain constant 215 for the duration of a transmission packet denoted by $T_{C}$ and 216 then they are faded independently from one packet to another

\footnotetext{
${ }^{4}$ In practice, this may be realized by a supercapacitor-based storage system, such as, for example, the Everlast solar system introduced in [1].

${ }^{5}$ In practice, the charging efficiency of the secondary EB may not reach $100 \%$; hence, it may be multiplied by an efficiency factor $\eta_{\text {buffer }} \in[0,1]$, which may be equivalently considered to be a reduced energy arrival rate, and hence, it does not affect any of our analysis.

${ }^{6}$ In practice, the power consumption of the circuits may be nonnegligible. We may assume that the harvesting system is capable of providing sufficient circuit power, while additionally providing a nonnegative transmit power. When the EH system is not capable of supplying sufficient circuit power, the transmitter may be switched off. On the other hand, the energy conversion efficiency $\eta_{\mathrm{TX}}$ from the EB to the transmitter cannot reach $100 \%$ in practice. Hence, we may simply multiply the energy arrival rate at the transmitter with an efficiency coefficient $\eta_{\mathrm{TX}} \in[0,1]$, which does not affect any of our analysis.
}

over the time dimension. Note that we make no assumptions 217 concerning the specific channel model and the distribution of 218 the channel gain. We also assume that there are always data 219 packets buffered at the $\mathrm{SN}$ for transmission. The signal received 220 at the DN is represented by

$$
y=h \sqrt{P_{t}} G_{s d} x+n
$$

where $h$ is the channel coefficient capturing the effects of fad- 222 ing, while $P_{t}$ is the transmit power, $x$ is the transmitted signal, 223 and $n$ is the additive noise at the receiver, which is modeled by 224 independent standard circularly symmetric complex Gaussian 225 random variables having a zero mean and a variance of 1. In (1), 226 the average processing gain of $G_{s d}=\left(N_{0} \times d_{s d}^{\beta}\right)^{-1}$ between 227 the $\mathrm{SN}$ and the DN captures the effect of both the pathloss 228 and the noise, where $N_{0}$ is the noise power at the receiver, $d_{s d}$ is 229 the distance between the $\mathrm{SN}$ and the DN, while $\beta$ is the pathloss 230 exponent.

231

An outage is defined as the event when the instantaneous 232 received signal-to-noise power ratio (SNR) $\gamma$ at the receiver 233 is below a predefined threshold $\gamma_{\text {th }}$ that has to be exceeded 234 for successful decoding. If idealized perfect capacity-achieving 235 coding is assumed, we have $\gamma_{\text {th }}=2^{R}-1$, where $R$ is the 236 data transmission rate [16]. Then, the OP of the single-hop EH 237 network may be expressed as follows:

$$
\begin{aligned}
P_{\text {out }} & =\operatorname{Pr}\left\{P_{t}|h|^{2} G_{s d}<\gamma_{\text {th }}\right\} \\
& \triangleq \operatorname{Pr}\left\{P_{t}|h|^{2}<P_{\text {th }}\right\}
\end{aligned}
$$

where $P_{t}$ is the transmit power, and $h$ is the normalized channel 239 coefficient capturing the fading effects. In (2), we define $P_{\text {th }}=240$ $\gamma_{\mathrm{th}} / G_{s d}$, to focus our attention on the effects of both the 241 transmit power $P_{t}$ and the channel's fading coefficient $h . \quad 242$

In the conventional transmission scheme relying on classic 243 constant power supply, the transmit power $P_{t}$ is a constant, and 244 the corresponding OP of narrow-band block-fading channels 245 was quantified in [16]. However, in the EH networks, the instan- 246 taneous transmit power $P_{t}$ is time variant, which is constrained 247 by the amount of the energy available in the EB, which in turn 248 is a random variable depending on the energy arrival rate. The 249 energy arrival rate is assumed to exhibit a blockwise fluctuating 250 nature, which remains constant over a TS of duration $T_{E}$ and 251 changes independently over the subsequent TSs. During a TS 252 with a duration of $T_{E}$, the amount of energy harvested, i.e., 253 $P_{\text {in }} T_{E}$, is independent of both that harvested in the previous TS 254 and of the energy consumed, i.e., $P_{t} T_{E}$, during transmission, 255 which is determined by the EB state $B_{T}$ at the beginning of the 256 current TS.

We define the EB state as $B_{T}=B_{E} / T_{E}$, where $B_{E}$ is the 258 amount of energy available in the EB, while $T_{E}$ is the duration 259 of the recharge cycle. The physical interpretation of $B_{T}$ is the 260 maximum average transmit power that may be supported by 261 the amount of energy stored in the buffer during the current 262 recharge cycle. ${ }^{7}$ The EH-causality constraint [2] is interpreted 263

\footnotetext{
${ }^{7}$ When the knowledge of the instantaneous CSI during a period is unavailable at the transmitter, transmitting at a constant transmit power would achieve the minimum OP [16]. Therefore, a constant transmit power is adopted during each recharge cycle, and $B_{T}$ is the upper bound.
} 


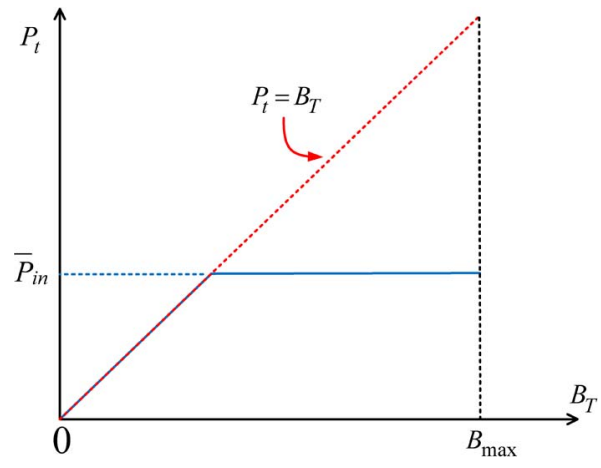

Fig. 2. EUP illustrated as the function of $P_{t}$ versus $B_{T}$.

264 as follows: the instantaneous transmit power $P_{t}$ cannot exceed 265 the maximum power $B_{T}$ that may be supported by the current $266 \mathrm{~EB}$ state, i.e., we have $P_{t} \leq B_{T}$, explicitly indicating that the 267 energy assigned for transmission must not exceed the amount 268 of energy harvested. We may model the EUP by the transmit 269 power as a function of the EB state, as follows:

$$
P_{t}\left(B_{t}\right), B_{T} \in\left[0, B_{\max }\right]
$$

270 where the EB state $B_{T}$ is upper bound by $B_{\max }$ defined as the $271 \mathrm{~EB}$ capacity divided by the recharge cycle $T_{E}$.

272 In Fig. 2, the EH-causality constraint is shown in dashed 273 lines as $P_{t}\left(B_{T}\right)=B_{T}$, which models the best-effort policy 274 proposed in [6], where all harvested energy in the buffer is 275 used up for transmission. On the other hand, the asymptotic 276 optimal policy proposed in [7] is illustrated by the solid line in 277 Fig. 2, where the SN aims to transmit at a power of $P_{t}=\bar{P}_{\text {in }}$. 278 In the asymptotic optimal policy, when the remaining energy in 279 the EB is capable of supporting a higher transmit power than 280 the average energy arrival rate $\bar{P}_{\text {in }}$, the transmitter conserves 281 the energy for its future usage. If the remaining energy in the 282 EB is insufficient for supporting $P_{t}=\bar{P}_{\text {in }}$, the SN switches to 283 the best-effort policy. We may formulate the OP of the P2P-EH 284 network as follows:

$$
\begin{aligned}
P_{\text {out }}\left(P_{t}\right) & =\int_{0}^{B_{\max }} \operatorname{Pr}\left\{P_{t}(x)|h|^{2}<P_{\text {th }}\right\} f_{B_{T}}(x) d x \\
& =\int_{0}^{B_{\max }} \int_{0}^{+\infty} \operatorname{Pr}\left\{P_{t}(x) y<P_{\text {th }}\right\} f_{|h|^{2}}(y) f_{B_{T}}(x) d x
\end{aligned}
$$

285 where $h$ is the channel coefficient capturing the effects of 286 fading, which is a random variable, and its $\operatorname{PDF} f_{|h|^{2}}(y)$ relies 287 on the statistical channel model. $f_{B_{T}}(x), x \in\left[0, B_{\max }\right]$ is the $288 \mathrm{PDF}$ of the EB state $B_{T}$. Therefore, to derive the OP formulated 289 in (4), the PDF of the EB state $B_{T}$ has to be modeled, bearing in 290 mind the specific EUP adopted. Furthermore, because both $P_{t}$ 291 and $B_{T}$ are continuous variables, the number of feasible EUPs 292 is infinite, and since different policies would result in different 293 EB-state PDFs, finding the optimal policy for minimizing the 294 OP in (4) may be quite challenging. Hence, we will investigate 295 this problem in the next section.
B. DMC Modeling of the EB State

As the energy arrival rate $P_{\text {in }}$ is assumed to be constant over 297 a recharge cycle $T_{E}$ and then changes independently over the 298 subsequent recharge cycles, the EB state $B_{T}(k)$ at the end 299 of the $k$ th $(k \geq 1)$ recharge cycle relies only on the state of 300 $B_{T}(k-1)$, on the amount of energy consumed for transmis- 301 sion $P_{t}\left[B_{T}(k)\right]$, as well as on the current energy arrival rate $P_{\mathrm{in}}, 302$ which obeys a certain PDF, but it is statistically independent 303 of its previous samples. Therefore, $B_{T}$ may be modeled by a 304 continuous Markov process.

305

However, the domain of $B_{T} \in\left[0, B_{\max }\right]$ is continuous; 306 hence, the set of the states is uncountable and challenging to 307 manage [17]. Therefore, given the EUP, deriving the PDF of $B_{T} 308$ is quite challenging, except for certain special cases, such as the 309 best-effort policy combined with the condition, when the trans- 310 mit power is equal to the instantaneous arriving energy, which 311 may be modeled by the exponential distribution [6]. Even for 312 the asymptotic optimal policy [7], where $P_{t}$ is a simple function 313 determined by a combination of the best-effort policy and of the 314 constant power supply, the PDF of $B_{T}$ cannot be readily derived 315 in closed form; hence, the asymptotic optimality relies on the 316 fact that the probability of $\operatorname{Pr}\left\{B_{T}<P_{t}=\bar{P}_{\text {in }}\right\} \rightarrow 0$, when the 317 EB size obeys $B_{\max } \rightarrow \infty$. In order to quantify and then to 318 minimize the OP in (4), we approximate the continuous-state 319 Markov process by a finite-state Markov chain [18], to model 320 the EB state $B_{T}$, and to derive the PDF of $B_{T}$. Specifically, the 321 EB size $B_{\max }$ is discretized as $L_{\max }=\left\lfloor B_{\max } / \varepsilon_{P}\right\rfloor$, where $\varepsilon_{P} 322$ is the discrete step size of the power. Therefore, $l=\left\lfloor B_{T} / \varepsilon_{P}\right\rfloor 323$ may take a value from $l \in\left\{0,1, \ldots, L_{\max }\right\}$ and has a state- 324 space size of $\left(L_{\max }+1\right)$. The instantaneous $\mathrm{EH}$ rate $P_{\text {in }}$ and 325 the decoding threshold $P_{\text {th }}$ are also discretized with a step size 326 of $\varepsilon_{P}$ as

$$
\begin{aligned}
L_{\mathrm{in}} & =\left\lfloor\frac{P_{\mathrm{in}}}{\varepsilon_{P}}\right\rfloor \\
L_{\mathrm{th}} & =\left\lfloor\frac{P_{\mathrm{th}}}{\varepsilon_{P}}\right\rfloor .
\end{aligned}
$$

Hence, $L_{\text {th }}$ is a discrete constant when $P_{\text {th }}$ is given, while $l$ and 328 $L_{\text {in }}$ are discrete random variables, and their probability mass 329 functions (PMFs) may be generated from the PDFs of $B_{T}$ and 330 $P_{\text {in }}$ as follows:

$$
\begin{gathered}
\operatorname{Pr}\{l=x\}=\int_{x \varepsilon_{P}}^{(x+1) \varepsilon_{P}} f_{B_{T}}(u) d u \\
\operatorname{Pr}\left\{L_{\text {in }}=x\right\}=\int_{x \varepsilon_{P}}^{(x+1) \varepsilon_{P}} f_{P_{\text {in }}}(u) d u .
\end{gathered}
$$

Although the variables $B_{T}, P_{\text {in }}$, and $P_{t}$ may assume any arbi- 332 trary continuous nonnegative value, the DMC may be capable 333 of sufficiently accurately capturing the buffer's behavior, as 334 long as the discretization step size $\varepsilon_{P}$ is small enough. Finally, 335 we may discretize the EUP formulated in (3) as

$$
P_{t}(l)=P_{t}\left(\left\lfloor\frac{B_{T}}{\varepsilon_{P}}\right\rfloor\right), l \in\left\{0,1, \ldots, L_{\max }\right\}
$$


337 where the discrete EUP is defined as

$$
L_{t}(l)=\left\lfloor\frac{P_{t}(l)}{\varepsilon_{P}}\right\rfloor .
$$

338 Then, we may construct the state transition matrix $T$ of the EB 339 states, where the specific element in the $i$ th row and $j$ th column 340 is given by

$$
\begin{aligned}
T_{i, j} & =\operatorname{Pr}\{l(k+1)=j \mid l(k)=i\} \\
& = \begin{cases}\operatorname{Pr}\left\{j=i+L_{\text {in }}-L_{t}(i)\right\}, & 0 \leq j<L_{\text {max }} \\
\operatorname{Pr}\left\{j \leq i+L_{\text {in }}-L_{t}(i)\right\}, & j=L_{\text {max }} .\end{cases}
\end{aligned}
$$

341 We arrive at the steady-state probability vector $\pi=\left[\pi_{0} \pi_{1} \ldots\right.$ $\left.342 \pi_{L_{\max }}\right]^{T}$ using the relationship of

$$
\pi=T^{T} \pi
$$

343 where the physical interpretation of (10) is that the state proba344 bility vector $\pi$ converges and remains constant. Then, we may 345 formulate the OP as

$$
\begin{aligned}
P_{\text {out }}\left(L_{t}(l)\right) & =\sum_{l=0}^{L_{\max }} \operatorname{Pr}\left\{L_{t}(l)|h|^{2}<L_{\text {th }}\right\} \pi(l) \\
& \triangleq \sum_{l=0}^{L_{\max }} P_{e}(l) \pi(l)
\end{aligned}
$$

346 which is the discrete version of (4). It should be noted that, 347 in (11), the OP component of $P_{e}(l) \triangleq \operatorname{Pr}\left\{L_{t}(l)|h|^{2}<L_{\mathrm{th}}\right\}$ is 348 not determined unambiguously by the EUP defined by $L_{t}(l)$, $349 l \in\left[0, L_{\max }\right]$, because it also relies on the statistical channel 350 model determining the distribution of $|h|^{2}$. For example, if a 351 narrow-band Rayleigh block-fading channel is assumed, then $352|h|^{2}$ follows the exponential distribution in conjunction with the 353 parameter of 1 . In this case, the $\mathrm{OP}$ component $P_{e}(l)$ may be 354 expressed as

$$
P_{e}(l)=\operatorname{Pr}\left\{L_{t}(l)|h|^{2}<L_{\mathrm{th}}\right\}=1-e^{-\frac{L_{\mathrm{th}}}{L_{t}(l)}} .
$$

\section{C. Two-Dimensional EUP-Search Algorithm}

356 Given a certain EUP represented by $L_{t}(l), l \in\left[0, L_{\max }\right]$ and 357 a specific statistical channel model, we are now capable of 358 quantifying the OP of a certain EUP with the aid of (7)-(11). 359 The optimal EUP $L_{t}(l), l \in\left[0, L_{\max }\right]$ may be formulated by 360 using the physically meaningful OF minimizing the OP as 361 follows:

$$
\min _{L_{t}(l)} P_{\text {out }}\left[L_{t}(l)\right]
$$

362 However, the inverse of the mapping in (11) from the OP $363 P_{\text {out }}\left[L_{t}(l)\right]$ to the specific EUP $L_{t}(l)$ cannot be readily evalu364 ated. In other words, given a certain $P_{\text {out }}[L(l)]$, it is not possible 365 to derive the EUP $L_{t}(l)$ adopted. Naturally, this hinders the related inverse mapping, and hence, the closed-form derivation 366 of the optimal EUP is not possible. Although the buffer-state 367 transition matrix $T$ of (9) may be readily determined, given 368 the EUP $L_{t}(l)$, according to (9), the resultant steady-state 369 probability vector $\pi=\left[\begin{array}{llll}\pi_{0} & \pi_{1} & \ldots & \pi_{L_{\max }}\end{array}\right]^{T}$ is a solution of 370 (10), which is a high-dimensional system of linear equations. 371 Furthermore, given a certain steady-state probability vector $\pi, 372$ it is not possible to derive the buffer-state transition matrix $T, 373$ and hence, we cannot uniquely and unambiguously determine 374 the discrete EUP $L_{t}(l)$.

1) Design Motivations: When using a discrete Markov mod- 376 eling of the EB state, the EUP is represented by a vector of 377 $L_{t}(l), l \in\left[0, L_{\max }\right]$, which has $\left(L_{\max }+1\right)$ legitimate elements 378 over the first dimension constituted by the EB state, where the 379 $l$ th element in $L_{t}(l)$ itself may be assigned any discrete value 380 spanning from 0 to $l$ over the second dimension representing the 381 amount of energy assigned for transmissions. Hence, the EUP 382 search is over a 2-D space. The aforementioned fact motivates 383 us to design an EUP-search algorithm. The most conceptually 384 straightforward way of finding the optimal EUP $L_{t}(l), l \in 385$ $\left[0, L_{\max }\right]$ is to invoke an exhaustive search, which evaluates 386 every feasible EUP and selects the one having the minimum 387 OP. As illustrated in Fig. 2, an EUP $L_{t}(l)$ is physically feasible 388 as long as the instantaneous transmit power $P_{t}$ is nonnegative 389 and does not exceed the maximum affordable power $B_{T}$ that 390 may be supported by the current EB state $P_{t} \leq B_{T}$, which is 391 equivalent to the following discrete form:

$$
0 \leq L_{t}(l) \leq l, \forall l \in\left[0, L_{\max }\right]
$$

This simple feasibility constraint results in a large num- 393 ber of feasible EUPs, where the complexity of searching for 394 the optimal policy that minimizes the OP may be excessive. 395 Quantitatively, there are $N_{f}=\left(L_{\max }+1\right)$ ! number of feasible 396 functions of $L_{t}(l)$, given the condition in (14). For example, if 397 we have $L_{\max }>11$, the number of feasible functions becomes 398 $N_{f}>10^{8}$. Therefore, the exhaustive search method of finding 399 the optimal policy is not practically feasible. Hence, we have 400 to design search algorithms having a practically tolerable com- 401 plexity, which are detailed in the following sections. 402

2) EUP-Search Algorithm Design: In the algorithms pro- 403 posed in this treatise, the design guidelines that we adopted for 404 controlling the complexity, which is quantified by the number 405 of $O P$ evaluations, are summarized as follows.

- Guideline 1: The optimal EUP $L_{t}(l), l \in\left[0, L_{\max }\right]$ is a 407 nondecreasing function of the EB state l, i.e., we have 408 $\forall k \in\left[0, L_{\max }-1\right], L_{t}(k+1)-L_{t}(k) \geq 0$. The physi- 409 cal interpretation of this guideline can be summarized as 410 follows. If the amount of energy available in the EB is 411 increased, the transmitter should not use a lower transmit 412 power. The reason behind this guideline is twofold: First, 413 the transmitter has no knowledge of the energy arrival rate 414 in the future; therefore; it cannot decide as to whether 415 conserving the harvested energy in the EB for future usage 416 is beneficial. Second, the transmitter has no knowledge of 417 the instantaneous channel gain; therefore, it cannot decide 418 how to control the transmit power. 
441 Although the aforementioned pair of design guidelines may 442 be interpreted physically in a simple manner, it is challeng443 ing to rigorously prove the optimality of Guideline 1, while 444 Guideline 2 is applied in a relatively high transmit power 445 scenario associated with a good channel quality, when the OP 446 is a convex function of the transmit power [8]. When relying 447 on the proposed pair of design guidelines, the number of OP 448 evaluations is reduced from $N_{f}=\left(L_{\max }\right)$ ! to $N_{2 D}=2^{N_{\max }}$, 449 which may still be excessive. Quantitatively, when we have $450 N_{\max }>30$, the number of OF evaluations obeys $N_{2 D}>10^{9}$. 451 Therefore, we conceive a third guideline for controlling the 452 complexity, albeit this is achieved at the cost of potentially 453 resulting in a locally optimal solution, which is detailed as 454 follows.

462 Since Guideline 3 may result in locally optimal solutions, 463 multiple initial solutions may be chosen for the search al464 gorithm. However, through our extensive numerical evalua465 tions conducted for $N_{\max }<12$, when the exhaustive search 466 algorithm is still feasible, our numerical results have shown 467 that Algorithm 1 is capable of finding the globally optimal 468 EUP. Algorithm 1 uses the best-effort policy as the initial 469 solution, and then, the three aforementioned guidelines are 470 followed throughout the rest of the design. Therefore, it may 471 be concluded that, although the optimality may not be shown 472 mathematically, the proposed heuristic 2D-search algorithms 473 are effective in practical applications, while imposing a much 474 lower complexity than the exhaustive search.

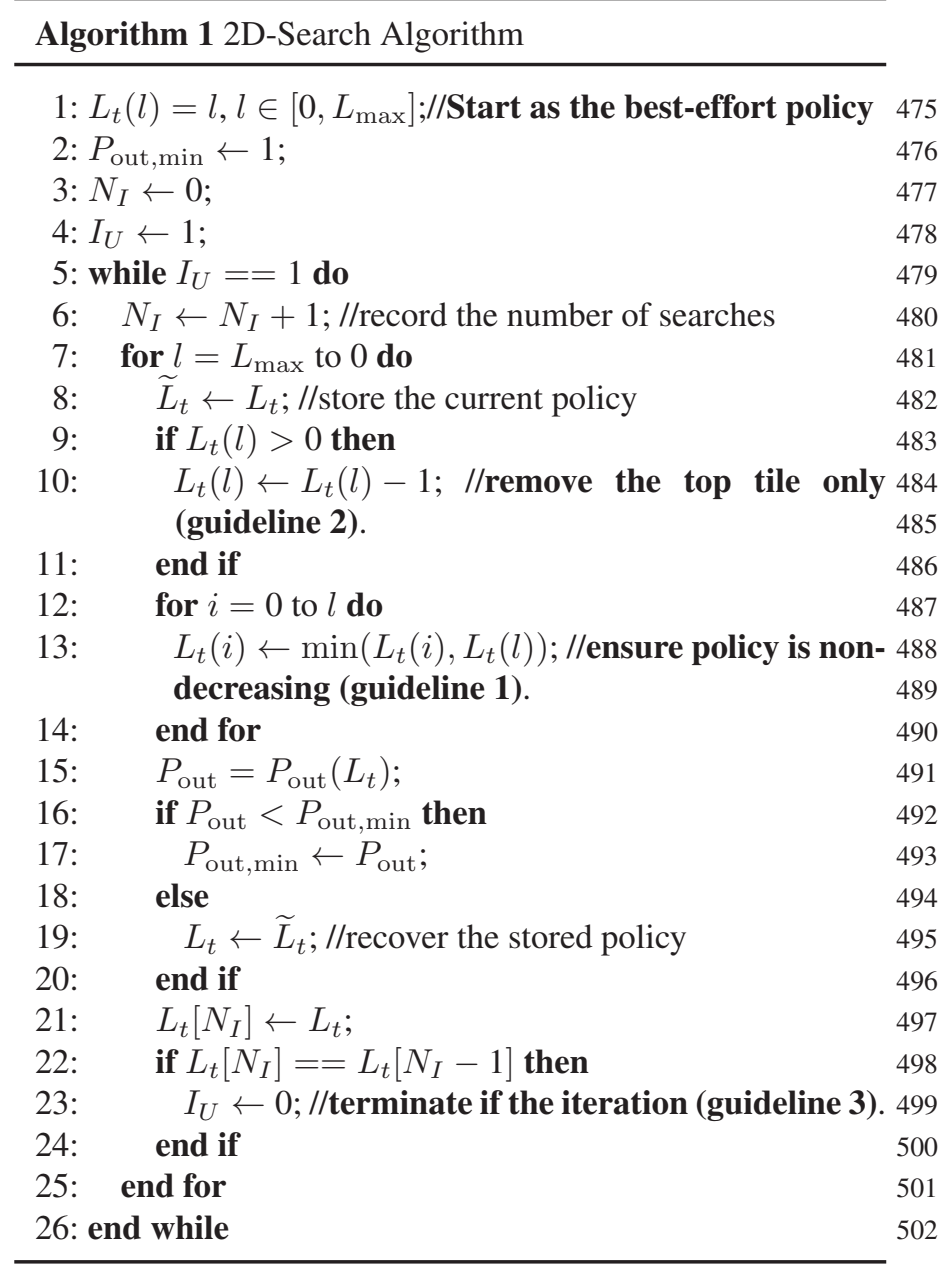

\section{One-Dimensional EUP-Search Algorithm}

In the previous section, the optimal EUP was investigated and 504 a 2D-search algorithm was proposed. However, the algorithm 505 relies on searching in a 2-D domain of the EB state and of the 506 energy assigned for transmission; hence, it is quite involved. 507 Here, motivated by the fact that the asymptotic optimal policy 508 is characterized by a constant desired transmit power [7], we 509 formulate a 1D-search-based EUP and aim for minimizing the 510 OP using a reduced-complexity 1-D search to exhibit a signifi- 511 cantly lower complexity than that of the 2D-search algorithm. 512

1) Design Motivations: Our proposed 1D-search policy is 513 motivated by the asymptotic optimal policy proposed in [7], 514 which is illustrated in Fig. 2. The suboptimal EUP considered 515 is based on a combination of the constant power policy and the 516 best-effort policy. Specifically, given a desired constant transmit 517 power $P_{d}$, when the energy remaining in the EB satisfies 518 $B_{t} \geq P_{d}$, the transmitter opts for transmitting at a power of 519 $P_{t}=P_{d}$ and conserves the rest of the energy for its future 520 usage. Otherwise, when $B_{t}<P_{d}$, the transmitter switches to 521 the best-effort policy and transmits at a power of $P_{t}=B_{T}$. The 522 suboptimal policy is represented by a fixed $P_{t}\left(B_{T}\right)$ of

$$
P_{t}\left(B_{T}\right)= \begin{cases}B_{T}, & B_{T}<P_{d} \\ P_{d}, & B_{T} \geq P_{d}\end{cases}
$$


524 while its discrete version represented by $L_{t}(l), l \in\left[0, L_{\max }\right]$ is

$$
L_{t}(l)= \begin{cases}l, & l<L_{d} \\ L_{d}, & l \geq L_{d}\end{cases}
$$

525 where we define $L_{d}=\left\lfloor P_{d} / \varepsilon_{P}\right\rfloor$. Compared to the generalized 526 representation of $L_{t}(l), l \in\left[0, L_{\max }\right]$, which requires $\left(L_{\max }+\right.$ 527 1) variables for fully characterizing the policy, the proposed 528 EUP may be characterized by a single variable $L_{d}$. Therefore, $529 L_{d}$ is also the only variable that may be optimized to minimize 530 the OP. However, the 1D-search policy may be expected to 531 result in a degraded OP.

532 A special case of the proposed EUP is to set $P_{d}=\bar{P}_{\text {in }}$ or 533 equivalently $L_{d}=\bar{L}_{\text {in }}$. The asymptotic optimal EUP proposed 534 in [7] was shown to achieve the performance of its constant535 power counterpart operating at $P_{t}=\bar{P}_{\text {in }}$, based on the assump536 tion of an infinite EB size of $B_{\max } \rightarrow \infty$ [7]. In this case, 537 the probability of an EB overflow is 0 , and the probability 538 of $\operatorname{Pr}\left\{B_{T}<P_{d}\right\}=\operatorname{Pr}\left\{l<L_{d}\right\} \rightarrow 0$. It is plausible that the 539 performance of the classic non-EH system constitutes the OP 540 lower bound that may be achieved by any EH system relying 541 on a random energy arrival rate. Naturally, achieving the per542 formance of the asymptotic optimal EUP is desirable [7].

543 However, when the EB size is finite, the asymptotic optimal 544 policy would be suboptimal, because a finite EB may overflow 545 with a nonnegligible probability, when the instantaneous energy 546 arrival rate is high and cannot be stored for future usage. 547 Meanwhile, the choice of $L_{d}=\bar{L}_{\text {in }}$ may not be optimal, since 548 a choice of $L_{d} \neq \bar{L}_{\text {in }}$ may reduce both the probability of EB 549 overflow and the OP. However, the optimal choice ${ }^{8}$ of $P_{d}$ is 550 not obvious, because the relationship between the OP $P_{\text {out }}$ and 551 the energy usage function $L_{t}$ is quantified by (9)-(11), which 552 makes the direct derivation of the optimal $P_{d}$ quite challenging. 553 By comparison, as shown in (7)-(11), given a specific value 554 of $P_{d}$, the numerical evaluation of $P_{\text {out }}$ may be straightforward, 555 according to the OP expression provided in (11). This motivates 556 us to design a search algorithm, which searches for the optimal $557 P_{d}$ based on the numerical evaluation of $P_{\text {out }}$, instead of using 558 an analytical derivation to get the optimal $P_{d}$ directly.

559 In the next section, we will first derive the OP for the 1D560 search-based EUP given a specific $L_{d}$ and then propose our 561 specific search algorithm for finding the optimal $L_{d}$ to minimize 562 the OP.

563 2) One-Dimensional EUP-Search Algorithm Design: Upon 564 invoking the 1D-search-based EUP represented in (16), we may 565 simplify the OP expression of (11) specifically for the 1D566 search policy as follows:

$$
\begin{aligned}
P_{\text {out }}=\operatorname{Pr}\{l & \left.\geq L_{d}\right\} \operatorname{Pr}\left\{L_{d}|h|^{2}<L_{\text {th }}\right\} \\
& +\operatorname{Pr}\left\{l<L_{d}\right\} \operatorname{Pr}\left\{l|h|^{2}<L_{\text {th }} \mid l<L_{d}\right\}
\end{aligned}
$$

567 where the first line represents the OP, when the energy in the $568 \mathrm{~EB}$ is capable of supporting transmitting at the desired level of $569 L_{d}$. The second line in (17) represents the OP, when the energy 570 in the EB is insufficient for transmitting at the power level of

\footnotetext{
${ }^{8}$ The optimal choice is in the context of selecting $P_{d}$ for the 1D-search algorithm, which may still result in inferior OP compared to the exhaustive search and the 2D-search algorithms.
}

$L_{t}=L_{d}$, and the transmitter consumes all the energy in the 571 $\mathrm{EB}$, while transmitting at a power level of $L_{t}=l$. Then, we 572 construct the state transition matrix $T$ of the EB state according 573 to (9), and when the EB state is steady, the state probability 574 vector $\pi$ may be formulated as follows:

$$
\pi=T^{T} \pi
$$

where $\pi=\left[\begin{array}{llll}\pi_{0} & \pi_{1} & \ldots & \pi_{L_{\max }}\end{array}\right]^{T}$. Given the desired power level 576 represented by $L_{d}$ and the OP expression in (17), we have 577

$$
\operatorname{Pr}\left\{l \geq L_{d}\right\}=\sum_{l=L_{d}}^{L_{\max }} \pi_{l} .
$$

If we assume furthermore that the channel obeys Rayleigh 578 fading, the other terms in (17) can be derived as follows:

$$
\begin{aligned}
& \operatorname{Pr}\left\{L_{d}|h|^{2}<L_{\mathrm{th}}\right\}=1-\exp \left(-\frac{L_{\mathrm{th}}}{L_{d}}\right) \\
& \operatorname{Pr}\left\{l<L_{d}\right\} \operatorname{Pr}\left\{l|h|^{2}<L_{\mathrm{th}} \mid l<L_{d}\right\} \\
& =\sum_{l=0}^{L_{d}-1} \pi_{l} \operatorname{Pr}\left\{l|h|^{2}<L_{\mathrm{th}}\right\} \\
& =\sum_{l=0}^{L_{d}-1} \pi_{l}\left[1-\exp \left(-\frac{L_{\mathrm{th}}}{l}\right)\right] .
\end{aligned}
$$

By substituting the terms of (18)-(20) into (17), we may arrive 580 at the analytical OP for transmission over Rayleigh block- 581 fading channels in the P2P-EH network in Fig. 1. If a differ- 582 AQ3 ent statistical channel model is adopted, we may reformulate 583 (19) and (20), accordingly. Throughout this paper, we use the 584 Rayleigh block-fading channel as a case study, although our 585 proposed OP analysis and the search algorithms conceived for 586 OP minimization are sufficiently general for arbitrary channel 587 models. The effects of other wireless channel models will be 588 investigated in our future research.

589

Therefore, given a specific value of $L_{d}$, the numerical eval- 590 uation of $P_{\text {out }}$ is straightforward, according to the OP expres- 591 sion provided in (17). Since it relies on the single parameter 592 $L_{d}$, a 1-D EUP-search algorithm may be designed for finding 593 the optimal $L_{d}$, instead of searching over a 2-D EUP space, 594 as in Section II-C. This 1D-search procedure is detailed in 595 Algorithm 2, which is much simpler than the 2D-search algo- 596 rithm in Section II-C. Specifically, in Algorithm 2, there are a 597 total of $\left(L_{\max }+1\right)$ candidate EUPs, since we have $L_{d} \in\{0,1,598$ $\left.\ldots, L_{\max }\right\}$. For each candidate EUP, the OP is evaluated using 599 (17), where the one achieving the minimum OP is selected.

600

$\begin{array}{ll}\text { Algorithm } 2 \text { 1D-Search Algorithm } & 601 \\ \text { 1: } L_{d, \text { opt }} \leftarrow 0 ; & 602 \\ \text { 2: } P_{\text {out,min }} \leftarrow 1 ; & 603 \\ \text { 3: for } L_{d}=0 \text { to } L_{\max } \text { do } & 604 \\ \text { 4: } \quad P_{\text {out }}=P_{\text {out }}\left(L_{d}\right) ; & 605 \\ \text { 5: if } P_{\text {out }}<P_{\text {out,min }} \text { then } & 606 \\ \text { 6: } \quad P_{\text {out,min }} \leftarrow P_{\text {out }} ; & 607 \\ \text { 7: } \quad L_{d, \text { opt }} \leftarrow L_{d} ; & 608 \\ \text { 8: end if } & 609 \\ \text { 9: end for } & \end{array}$


610 Specifically, the 1-D EUP-search procedure of Algorithm 2 611 requires $\left(L_{\max }+1\right)$ evaluations of the $\mathrm{OP}$, which is signifi612 cantly lower than that of the 2-D EUP-search of Algorithm 1 613 or the exhaustive search methods. The low complexity of 614 Algorithm 2 accrues from the fact that the EUP functions $615 L_{t}(l)$ investigated may be characterized by a single scalar $L_{d}$, 616 as shown in (16). Therefore, the OP may be expressed as a 617 function of a scalar $L_{d}$, rather than as a vector $\overrightarrow{L_{t}} \triangleq\left\{L_{t}(l) \mid l \in\right.$ $\left.618\left[0, L_{\max }\right]\right\}$. In Section IV-A, we will compare the OP of the 619 proposed 2D-search and 1D-search Algorithms 1 and 2 to a pair 620 of state-of-the-art EUPs found in the literature, namely, to the 621 best-effort policy [6] and to the asymptotic optimal policy [7].

622

623

624 In the previous section, the EUPs conceived for minimizing 625 the OP of P2P networks were investigated. Here, we continue 626 by investigating the EUP design of an SDMA prototype net627 work. Compared to the P2P network, the outage events of 628 different EH-SNs are correlated, but a centralized optimization 629 would impose an excessive complexity. Even if a subopti630 mal 1-D EUP search space is adopted for each EH-SN, an $631 M$-dimensional search space is required for an SDMA network 632 of $M$ EH-SNs, which is generally not practical. In addition, the 633 global knowledge of the channel quality between each EH-SN 634 and the DN, as well as the statistical distribution of the energy 635 arrival rates, should be available at a central controller node, 636 which also imposes a high side-information signaling overhead 637 and complexity. Furthermore, for traditional non-EH SDMA 638 networks, the closed-form OP expressions are not available in 639 the open literature for generalized SDMA networks having $M$ $640 \mathrm{SNs}$, since the derivation of the closed-form OP expressions for 641 SDMA-EH networks is quite challenging.

642 Therefore, we embark on the OP analysis of an SDMA 643 network relying on ML detection and use the minimum-SNR 644 (min-SNR) approximations to arrive at the approximate OP 645 of our SDMA networks, which has been documented in [20] 646 and [21]. It will be shown that the min-SNR approximations 647 are accurate in predicting the OP of the SDMA networks. 648 Given an SDMA network comprised of $M$ EH-SNs and a $649 \mathrm{DN}$, we decompose the approximate joint OP of SDMA into 650 a product of $M$ mutually independent OP components, each of 651 which corresponds to a P2P-EH-network counterpart. Then, we 652 propose a DEUPO protocol, in which each EH-SN is capable of 653 optimizing its own EUP based on the 2-D and 1-D EUP-search 654 algorithms in Section II, using the statistics of its own uplink 655 (UL) channel and its own energy arrival rates, indicating that 656 only local knowledge is required.

\section{A. System Model and OP Formulation}

658 We consider a network of $(M+1)$ nodes, where $M$ SNs $659\left\{S_{m}, 1 \leq m \leq M\right\}$ transmit their individual information to a 660 common $\mathrm{DN}$, and each $\mathrm{SN}$ is equipped with both a harvesting 661 scheme and an EB, as shown in Fig. 1. Again, we assume 662 a narrow-band Rayleigh block-fading channel model, where 663 the fading coefficients remain constant for the duration of a

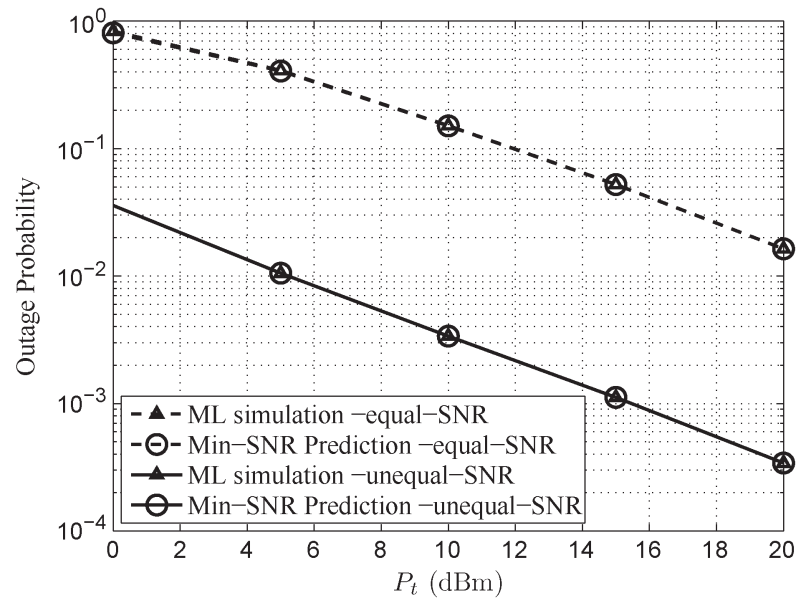

Fig. 3. Accuracy of the OP ( $\left.P_{\text {out }}\right)$ evaluation using the min-SNR approximations for $M=4, R=0.5 \mathrm{~b} / \mathrm{s} / \mathrm{Hz}$. The distance between the SNs and the DN is $d_{s d}=100 \mathrm{~m}$ and the pathloss exponent is $\beta=3$.

packet and then are faded independently from one packet to 664 another in both time and space. The additive noise imposed by 665 the receivers is modeled by independent zero-mean circularly 666 symmetric complex Gaussian random variables with a variance 667 of unity.

668

The DN is assumed to have perfect channel knowledge and 669 adopts ML detection. All the SNs transmit their messages 670 concurrently at the rate of $R$. The $\mathrm{SN} S_{m}$ encodes a bit 671 sequence into a codeword and transmits it to the DN, where 672 the DN jointly decodes the codewords received from all the 673 SNs. Therefore, the SN-DN hop may be modeled by a multiple- 674 access channel (MAC), and the criterion used for successful 675 decoding is

676

$$
\sum_{m \in S} R \leq \log \left(1+\sum_{m \in S} \gamma_{m d}\right) \forall S \subseteq\left\{S_{m}, 1 \leq m \leq M\right\}
$$

where $\gamma_{m d}$ represents the instantaneous received SNR of the 677 $S_{m}$-DN link. There are $(M !-1)$ inequalities in $(21)$, and even 678 if a single one of the inequalities in (21) is not satisfied, the 679 transmission over the SN-DN hop becomes erroneous. Hence, 680 when the min-SNR of the $M$ channels spanning from the 681 SNs to the reference node (RN), defined as $\gamma_{s d}^{\min }=\min _{m \in S} \gamma_{m d}$, $682 \quad \mathbf{A Q}$ is lower than the threshold $\gamma_{\mathrm{th}}^{s d}=2^{R}-1$ to be exceeded for 683 successful decoding, an outage event occurs. Therefore, we 684 aim for modeling the OP of the $M$-user MAC on the SN-RN 685 hop with the aid of the specific SN-RN link having the min- 686 SNR $\gamma_{s d}^{\min }$

Specifically, in Fig. 3, we compare the OP of the $M$-user 688 MAC channel using ML detection to that of a single link 689 having the min-SNR $\gamma_{s d}^{\min }$ of the $M$-user system. As shown 690 in Fig. 3, the OP of the two systems obtained by simulation 691 perfectly matches for both the equal-SNR and the unequal-SNR 692 scenarios. Specifically, in the equal-SNR situation, the average 693 channel quality of the link spanning from each SN to the DN 694 is identical, while in the unequal-SNR scenario, the average 695 channel quality is different, where the SNRs of the $M=4696$ links are one, two, four, and eight times higher than that in the 697 
698 equal-SNR situation, respectively. It is shown in Fig. 3 that 699 the exact OP of the $M$-user MAC channel and the predicted 700 OP using the P2P channel associated with the min-SNR are 701 identical for both scenarios. Hence, the OP using the min-SNR 702 approximation may be expressed as follows:

$$
\begin{aligned}
P_{\mathrm{out}, S D} & \approx \operatorname{Pr}\left\{\min _{m \in S} \gamma_{m d}<\gamma_{\mathrm{th}}^{s d}\right\}=1-\operatorname{Pr}\left\{\min _{m \in S} \gamma_{m d} \geq \gamma_{\mathrm{th}}^{s d}\right\} \\
& =1-\prod_{m \in S} \operatorname{Pr}\left\{\gamma_{m d}<\gamma_{\mathrm{th}}^{s d}\right\} \triangleq 1-\prod_{m \in S}\left(1-P_{\mathrm{out}, m d}\right) .
\end{aligned}
$$

\section{B. DEUPO Protocol}

704 Having confirmed the accuracy of the min-SNR approxima705 tion, we are now in the position to formulate the OP minimiza706 tion problem for the SDMA-EH network as follows:

$$
\min _{L_{t, 1}(l), L_{t, 2}(l), \ldots, L_{t, m}(l)} P_{\text {out }, S D}\left(L_{t, 1}(l), L_{t, 2}(l), \ldots, L_{t, m}(l)\right)
$$

707 where $L_{t, 1}(l), L_{t, 2}(l), \ldots, L_{t, m}(l)$ corresponds to the discrete 708 EUPs at the SNs. Equivalently, the minimization problem de709 fined in (23) may be expressed by

$$
\max _{L_{t, 1}(l), L_{t, 2}(l), \ldots, L_{t, m}(l)}\left[1-P_{\mathrm{out}, S D}\left(L_{t, 1}(l), L_{t, 2}(l), \ldots, L_{t, m}(l)\right)\right] .
$$

710 Let us now investigate the formulation of $[1-$ $\left.711 P_{\text {out }, S D}\left(L_{t, 1}(l), L_{t, 2}(l), \ldots, L_{t, m}(l)\right)\right]$ in detail. By using 712 the min-SNR approximation of (22), we have

$$
\begin{aligned}
& {\left[1-P_{\text {out }, S D}\left(L_{t, 1}(l), L_{t, 2}(l), \ldots, L_{t, m}(l)\right)\right]} \\
& \approx \prod_{m \in S}\left[1-P_{\text {out }, m d}\left(L_{t, m}(l)\right)\right] .
\end{aligned}
$$

713 In order to maximize the OF of (24), we may maximize each 714 component of $\left[1-P_{\text {out }, m d}\left(L_{t, m}(l)\right)\right]$. Since they are mutually 715 independent or equivalently, we may minimize each compo716 nent's $P_{\text {out }, m d}\left(L_{t, m}(l)\right)$. This is beneficial, because the $m$ th 717 component $P_{\text {out }, m d}\left(L_{t, m}(l)\right)$ corresponds to the OP of a P2P$718 \mathrm{EH}$ link spanning from the $m$ th EH-SN to the DN, while it is 719 independent of both the channel quality and the EUPs adopted 720 by other EH-SNs.

721 Therefore, we may design a DEUPO protocol, in which each 722 EH-SN optimizes its own EUP relying on the proposed 1D723 search and 2D-search algorithms proposed for a P2P link in 724 Section II. Specifically, we design the protocol as follows.

- Acquiring the Energy Arrival Rate and Channel Statistics: In practical applications, the system designer may choose appropriate EHI and CSI estimation algorithms, through which the system may detect the changes, generate a trigger, and decide when to activate its EUP optimization. This is a widely used event-triggered protocol [22], [23]. A simpler solution is to periodically invoke the EUP optimization, according to the instantaneous estimated statistics of both the energy arrival rates and the channels. This is, however, beyond the scope of this paper. Instead, we focus our attention on the issue of 735 deciding the EUP, whenever the optimization is activated. 736 In our analysis, we assume that both the estimated en- 737 ergy arrival rate and the channel statistics are perfectly 738 estimated. Hence, each EH-SN has perfect knowledge of 739 the statistics of energy arrival rate, while the DN has the 740 knowledge of the statistics of the UL channels spanning 741 from each EH-SN. In practice, this knowledge is acquired 742 with the aid of pilot-based channel estimation mechanism 743 and/or prediction methods.

744

- Local EUP Optimization Phase: Each SN sends a 745 request-to-send (RTS) packet to the DN. The DN would 746 send $M$ clear-to-send (CTS) packets to the $M$ SNs, where 747 the channel statistics between the $m$ th EH-SN and the DN 748 would be conveyed in each CTS packet, which is assumed 749 to be perfectly recovered at the EH-SNs. Then, each EH- 750 SN may adopt the 2D-search in Section II-C or the 1D- 751 search in Section II-D to find the approximate EUP for 752 our P2P-EH network. As discussed in the context of (25), 753 our design objective is to minimize the approximate OP 754 of the SDMA-EH network considered.

- Data Transmission Phase: Each EH-SN commences its 756 session, by transmitting to the DN, by relying on its 757 locally optimized EUP.

\section{Numerical Results}

A. P2P Networks

As detailed in Sections II-C and D, the OP relies on the 762 following system parameters:

- Statistics of the energy arrival rates: include the average 764 energy arrival rate $\bar{P}_{\text {in }}$ and the recharge cycle $T_{E}$. The 765 distribution of the fading energy arrival directly affects its 766 rate, which is assumed to be exponentially distributed, as 767 in [6] and [7], to facilitate our comparisons with the state- 768 of-the-art benchmarkers proposed in these references. $\quad 769$

- Statistics of the wireless information-transfer chan- 770 nels: again, the wireless channel spanning from the SN 771 to the DN is assumed to obey Rayleigh block fading, 772 although our analysis technique can be applied to arbitrary 773 channel models.

- Parameters of the EH-SN: the EB size $B_{\max }$ and the 775 data transmission rate $R$.

Here, the dependence of the OP on the aforementioned sys- 777 tem parameters will be investigated. In the context of the P2P- 778 EH networks, the distance between the SN and the DN is set 779 to $d_{s d}=100 \mathrm{~m}$ and the pathloss exponent to $\beta=3$, while the 780 noise power at the receiver is assumed to be $N_{0}=-80 \mathrm{dBm} .781$ The data transmission rate is set to $R=1 \mathrm{~b} / \mathrm{s} / \mathrm{Hz}$. In the figures, 782 the analytical results are represented by the dashed curves, 783 while the simulation results are shown by the symbols. It should 784 be noted that the discrete step sizes $\varepsilon_{P}$ used for quantifying the 785 OP and for searching for the feasible EUP sets are different. 786 For OP evaluations, $\varepsilon_{P}$ is set for ensuring that we have $L_{\max }=787$ 6400 to guarantee a high accuracy of quantifying the OP, while 788 we have $\varepsilon_{P}$ set to $L_{\max }=200$, when searching for the EUP 789 using Algorithms 1 and 2 to control the search complexity. 790 


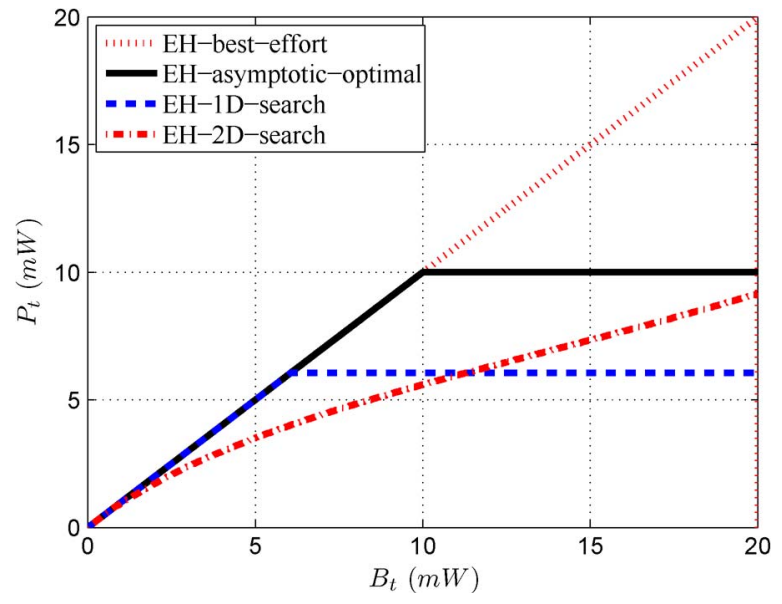

Fig. 4. Transmit power versus the EB state of different EUPs for the P2P network. The average energy arrival rate is $\bar{P}_{\mathrm{in}}=10 \mathrm{dBm}$, and the EB size is $B_{\max }=16 \bar{P}_{\text {in }}$, while we have $R=1 \mathrm{~b} / \mathrm{s} / \mathrm{Hz}$ and $T_{E}=8 T_{C}$.

791 We will demonstrate that the analytical results represented by 792 the dashed curves closely match the simulation results, which 793 indicates that the DMC-based analytical framework is capable 794 of accurately predicting the OP of the P2P-EH networks for all 795 of the EUPs considered.

796 The transmit power versus EB state of the different EUPs 797 are characterized in Fig. 4. It is shown that the best-effort 798 policy proposed in [6] exhibits a slope of 1, indicating that 799 the currently harvested amount of energy in the EB will be 800 immediately used up for transmission. The $x$-axis $B_{t}$ represents 801 the maximum power that may be supplied, given the amount of 802 energy in the EB for a period of $T_{E}$. The asymptotic optimal 803 policy is based on a combination of two trends: when the 804 amount of energy in the EB satisfies $B_{t}<\bar{P}_{\text {in }}$, the EH-SN 805 transmits by employing the best-effort EUP; otherwise, its EH$806 \mathrm{SN}$ opts for a constant power strategy by choosing a fixed 807 transmit power of $P_{t}=\bar{P}_{\text {in }}$. When the EB size tends to infinity, 808 the asymptotic optimal policy would approach the performance 809 of the constant power policy, indicating that a large EB is 810 capable of converting an EH system into an equivalent classic 811 non-EH system having a constant transmit power of $P_{t}=\bar{P}_{\text {in }}$ 812 [7]. However, when the EB size is finite, the asymptotic optimal 813 policy is no longer optimal in terms of minimizing the OP, as 814 shown in Fig. 5.

815 In Fig. 5, the performance of the EUPs found by the proposed 816 2D-search and 1D-search Algorithms 1 and 2 are compared 817 to that of the best-effort policy and the asymptotic optimal 818 policy proposed in [6] and [7], respectively. It is shown that, 819 for the given configurations, the OP achieved by the proposed 820 algorithms tends to be better than those achieved by the bench821 markers. Specifically, the 2D-search Algorithm 1 performs 822 close to its classic non-EH counterpart, which serves as the 823 lower bound of the OP for the EH systems [7]. At $P_{\text {out }}=0.01$, 824 the EUP found by the 2D-search Algorithm 1 achieves a 3-dB 825 power gain over the asymptotic optimal policy and a $6-\mathrm{dB}$ gain 826 over the best-effort policy. Therefore, if an EH-SN adopts the 827 asymptotic optimal policy, it requires twice the average energy 828 arrival rate harvested from the environment, compared with an 829 EH-SN equipped with the proposed 2D-search algorithm, while

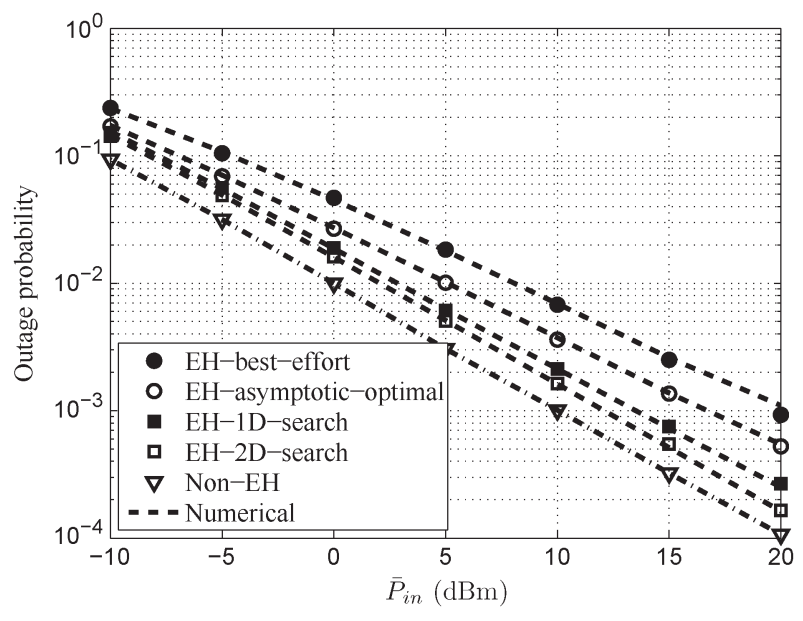

Fig. 5. OP versus average energy arrival rate $\bar{P}_{\text {in }}$ using different EUPs for the $\mathrm{P} 2 \mathrm{P}$ network. The EB size is $B_{\max }=16 \bar{P}_{\mathrm{in}}, R=1 \mathrm{~b} / \mathrm{s} / \mathrm{Hz}$, and $T_{E}=8 T_{C}$. The numerical OP results were evaluated from (11), given the searched EUP.

maintaining the same OP of $P_{\text {out }}=0.01$. This ratio would, 830 in fact, be further increased to four, if the benchmark EH-SN 831 adopts the best-effort policy.

We may conclude that the 2D-search algorithm is capable 833 of most significantly improving the EH-SN's capability to 834 exploit the harvested energy, or to substantially simplify the 835 hardware required for harvesting the energy from the envi- 836 ronment, which is important for applications such as WSNs 837 [1]. For example, the best-effort policy requires a four times 838 higher average energy arrival rate for maintaining an identical 839 outage performance as that using the 2D-search algorithm. 840 Equivalently, the amount of power harvested by the solar panel 841 increases linearly with the area of the solar panel [1], hence 842 requiring a four times larger solar panel. In other words, the 843 2D-search Algorithm 1 allows us to design a sensor node 844 having a solar panel of much smaller size, which has $25 \%$ of 845 the area necessitated by the best-effort policy. Furthermore, as 846 shown in Fig. 5, when the reliability requirements are more 847 stringent, the performance improvements of the proposed EUPs 848 would become more significant in terms of requiring a lower 849 energy arrival rate or a smaller solar panel. Finally, the 1D- 850 search Algorithm 2 is inferior to the 2D-search Algorithm 1, 851 since it exhibits a modest performance degradation of $0.9 \mathrm{~dB} 852$ at $P_{\text {out }}=10^{-2}$. From an alternative perspective, an EH-SN 853 adopting the 1D-search Algorithm 2 may require 1.23 times 854 higher energy arrival rate, which is the price paid for reducing 855 the computational complexity. Therefore, in a WSN application 856 scenario having sensor nodes that have a low computational 857 capability, the 1D-search Algorithm 2 or the simple asymptotic 858 optimal policy may be preferred.

The fundamental reason for the OP improvements of the 860 proposed 2D-search and 1D-search Algorithms 1 and 2 may be 861 inferred from Fig. 6, which represents the PMF of the discrete 862 EB state $l$ for different EUPs. It is observed that all EUPs 863 resulted in near-constant PMF values, apart from the peaks 864 at the states, when the EB was full at $l=L_{\max }$. Compared 865 with the PMF of the best effort and the asymptotic optimal 866 policy, the 2D-search and 1D-search Algorithms 1 and 2 may 867 


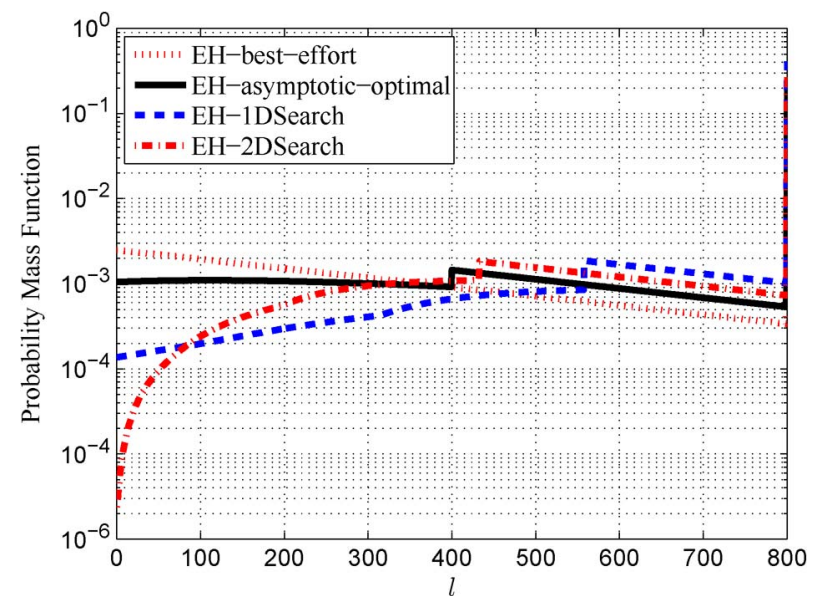

Fig. 6. PMF of the EB states for the P2P network. The average energy arrival rate is $\bar{P}_{\text {in }}=10 \mathrm{dBm}$, while the EB size is $B_{\max }=16 \bar{P}_{\text {in }}, R=1 \mathrm{~b} / \mathrm{s} / \mathrm{Hz}$, and $T_{E}=8 T_{C}$. The results were evaluated via simulations.

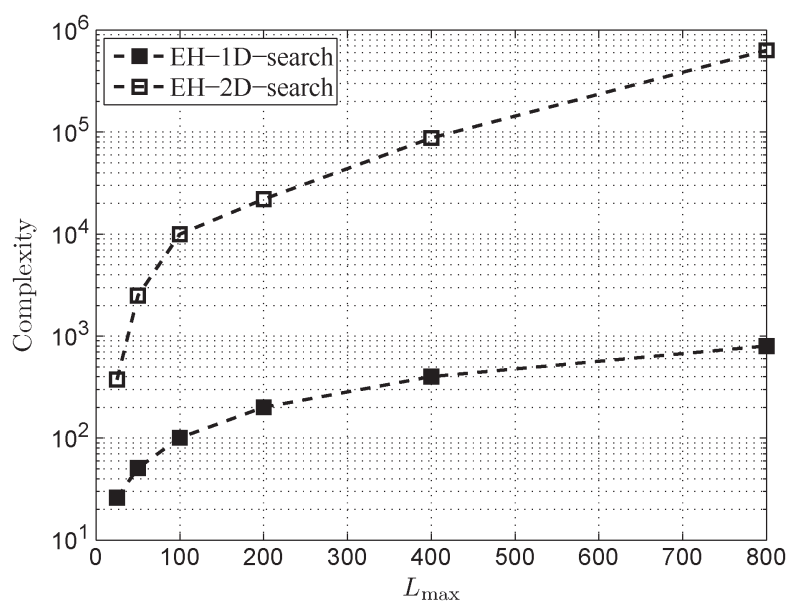

Fig. 7. Computational complexity, in terms of the number of OP evaluations versus the discrete $\mathrm{EB}$ size $L_{\max }$, for the $1 \mathrm{D}$-search and the $2 \mathrm{D}$-search algorithms for the P2P network. The results were evaluated via simulations.

868 be capable of improving the PMF when the EB is small, which 869 reduces the weights $\pi_{l}$ for the relatively large OP components 870 of $P_{e}(l)=\operatorname{Pr}\left\{L_{t}(l)|h|^{2}<L_{\mathrm{th}}\right\}$ in (11), when the discrete 871 transmit power $L_{t}$ is low. Therefore, reshaping the PMF by 872 reducing the contribution of the high OP components and 873 increasing the weights of the low OP components, the overall 874 OP may be beneficially reduced, which is confirmed by the 875 results in Fig. 5. It is also shown that the 1D-search Algorithm 2 876 may be capable of finding an EUP, which performs close to the 877 2D-search Algorithm 1, despite its lower complexity, as shown 878 in Fig. 7.

879 More explicitly, the relationship between the number of OP 880 evaluations and the discrete EB size $L_{\max }$ is illustrated in Fig. 7, 881 for both the 2D-search Algorithm 1 and 1D-search Algorithm 2. 882 Observe that the 1D-search Algorithm 2 drastically reduces the 883 complexity of its 2D-search counterparts. Quantitatively, when 884 the discrete EB size is $L_{\max }=400$, the 1D-search Algorithm 2 885 imposes as little as $0.46 \%$ of the computational complexity 886 compared with that of its 2D-search-based counterpart, while 887 imposing only a modest $0.9-\mathrm{dB}$ loss at $P_{\text {out }}=10^{-2}$, as shown

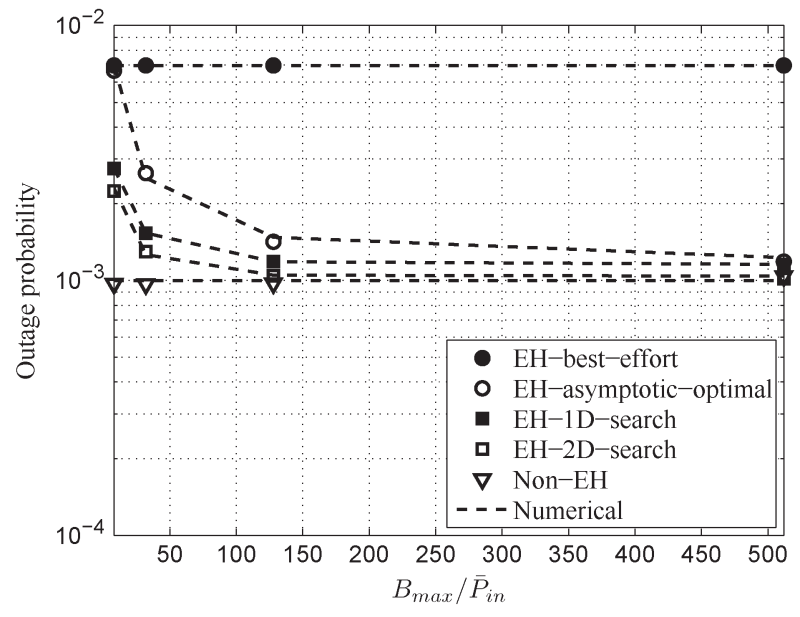

Fig. 8. OP versus the EB size $B_{\max }$ using different energy policies for the P2P network considered. The average energy arrival rate is $\bar{P}_{\text {in }}=10 \mathrm{dBm}$, $R=1 \mathrm{~b} / \mathrm{s} / \mathrm{Hz}$, and $T_{E}=8 T_{C}$. The numerical OP results were evaluated from (11), given the searched EUP.

in Fig. 5. On the other hand, from an overall energy con- 888 sumption point of view, the computation of the EUP also 889 dissipates a nonnegligible portion of the energy, particularly 890 for users relying on low-end devices. Therefore, the 1D-search 891 Algorithm 2 may be deemed attractive for applications relying 892 on hardware having a low computational capability, such as 893 mobile phones and wireless sensors.

894

In Fig. 8, the impact of EB size $B_{\max }$ is investigated. The 895 horizontal axis is $B_{\max } / \bar{P}_{\text {in }}$. It is shown in Fig. 8 that, when 896 the EB size increases, the OP of both the asymptotic optimal 897 policy proposed in [7] and the EUP relying on our 2D-search 898 Algorithm 1 improves, and they would converge to that of their 899 conventional non-EH counterparts. However, as the EB size 900 $B_{\max }$ increases, the EUP found by the 2D-search Algorithm 1901 may achieve a much better OP, when the EB size is small, 902 and it may converge to that of its classic non-EH counterpart. 903 This confirms the superiority of the proposed search algorithms 904 conceived for EH systems having a finite EB, particularly when 905 the available size of the EB is severely limited.

906

\section{B. Multiple-Access Networks}

907

In Fig. 9, the OP of our SDMA-EH network is investigated, 908 and the EUPs found by the proposed 2D-search and 1D-search 909 algorithms in Section II are compared with those of the best- 910 effort policy and asymptotic optimal policy. It is shown that, for 911 the given configurations, the OPs achieved by the proposed al- 912 gorithms are better than those of the benchmarks. Furthermore, 913 it is shown that the analytical results represented by dashed 914 curves closely match the simulation results, which indicates 915 that the proposed min-SNR approximation and the DMC-based 916 analytical framework are accurate.

Specifically, the 2D-search algorithm performs within $2 \mathrm{~dB} 918$ from its classic non-EH counterpart at $P_{\text {out }}=10^{-2}$, which 919 serves as the lower bound of the OP for EH systems [7]. 920 At $P_{\text {out }}=10^{-2}$, the EUP found by the 2D-search algorithm 921 achieves a 4.6-dB power gain compared with the asymptotic 922 optimal policy and an 8-dB power gain compared with the 923 


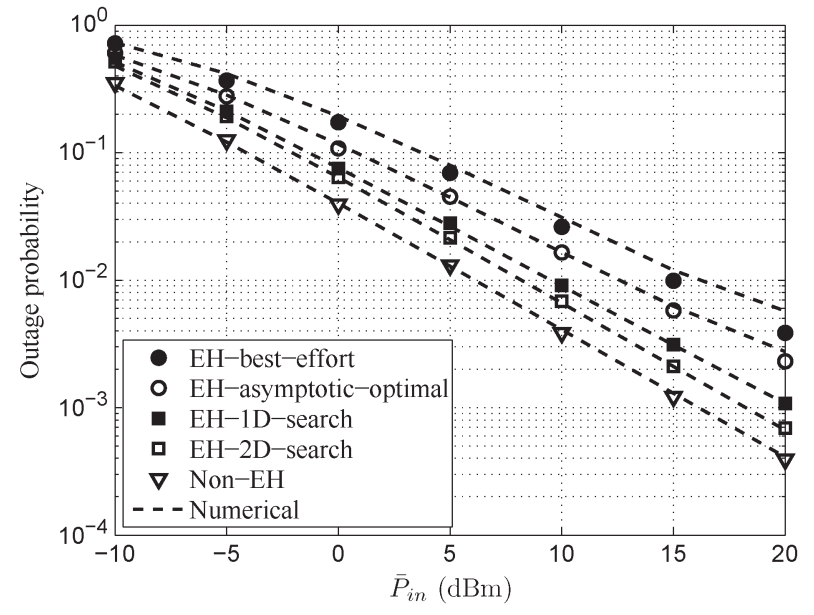

Fig. 9. OP versus average energy arrival rate $\bar{P}_{\text {in }}$ using different energy policies for the SDMA network associated with $M=4$ SNs. The EB size $B_{\max }=16 \bar{P}_{\text {in }}, R=1 \mathrm{~b} / \mathrm{s} / \mathrm{Hz}$, and $T_{E}=8 T_{C}$. The numerical OP results were evaluated from (22), given the searched EUP.

924 best-effort policy. Therefore, if an EH-SN adopts the asymp925 totic optimal EUP, it requires $10^{4.6 / 10} \approx 2.9$ times higher av926 erage energy arrival rates harvested from the environment, as 927 compared to an EH-SN equipped with the proposed 2D-search 928 algorithm at $P_{\text {out }}=10^{-2}$. This ratio would be further increased 929 to a factor of 6.3, if the benchmark EH-SN adopts the best930 effort policy. The 1D-search algorithm is suboptimal; hence, it 931 exhibits a performance degradation of $1.4 \mathrm{~dB}$ compared to that 932 of the 2D-search algorithm at $P_{\text {out }}=10^{-3}$. From a different 933 perspective, an EH-SN adopting our 1D-search algorithm may 934 require a 1.4 times higher energy arrival rate, which is the price 935 paid for its reduced computational complexity. In our future 936 work, we will jointly consider the optimization of the energy 937 arrival rate and of the power savings of the reduced-complexity 938 algorithms. This might, in fact, favor the 1-D algorithm over its 939 2-D counterpart.

940 Finally, we investigate the effects of the number of EH-SNs 941 on the OP in SDMA-EH networks. It is shown in Fig. 10 that, as 942 the number of SNs $M$ increases, the OP of all EUPs is reduced. 943 However, the EUPs found by the proposed 2D-search and 944 1D-search algorithms always outperform both the asymptotic 945 optimal policy and the best-effort policy. The results allow 946 the SDMA-EH network to accommodate more users, while 947 maintaining the same reliability. For example, if a maximum 948 OP of $P_{\text {out }}=10^{-2}$ is tolerable in the SDMA-EH network, 949 both the best-effort policy and the asymptotic policy may be 950 capable of supporting $K=2$ and 4 users, while the 1D-search 951 and the 2D-search algorithms support more than $K=8$ users 952 simultaneously. To conclude, given the proposed 1D-search and 953 2D-search algorithms, our receiver is capable of simultaneously 954 offering reliable services for significantly more EH users.

\section{CONCLUSION}

956 In this paper, we have summarized the state-of-the-art EUP 957 design aiming for minimizing the OP of P2P-EH networks 958 reported in the literature, and then, we have proposed two 959 novel algorithms, which are capable of exploiting the harvested

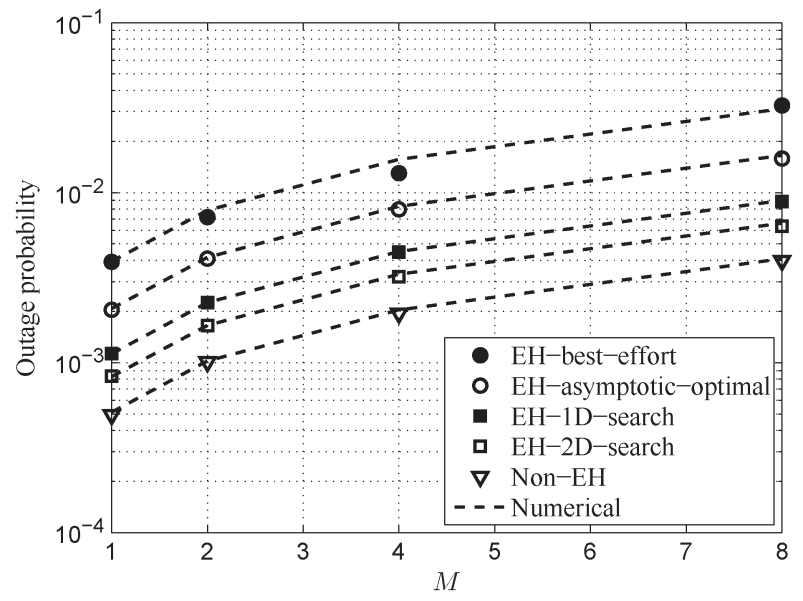

Fig. 10. OP versus average energy arrival rate $\bar{P}_{\text {in }}$ using different energy policies for the SDMA network associated with different number of SNs $M=1,2,4,8$. The average energy arrival rate is $\bar{P}_{\text {in }}=10 \mathrm{dBm}$, the EB size is $B_{\max }=16 \bar{P}_{\mathrm{in}}, R=1 \mathrm{~b} / \mathrm{s} / \mathrm{Hz}$, and $T_{E}=8 T_{C}$. The numerical OP results were evaluated from (22), given the searched EUP.

energy stored in a finite EB, where we showed that, using the 960 proposed algorithms, the achievable OP outperforms the state- 961 of-the-art benchmark systems found in the literature. Further- 962 more, upon invoking the proposed min-SNR approximation, the 963 algorithms advocated were invoked for SDMA-EH networks, 964 where we designed a DEUPO protocol. With the advent of the 965 DEUPO protocols proposed in this paper, our proposed 1D- and 966 2D-search algorithms require a significantly reduced energy 967 arrival rate at a given target $\mathrm{OP}$.

968

\section{REFERENCES}

[1] S. Sudevalayam and P. Kulkarni, "Energy harvesting sensor nodes: Sur- 970 vey and implications," IEEE Commun. Surveys Tuts., vol. 13, no. 3, 971 pp. 443-461, 3rd Quart. 2011.

[2] O. Ozel, K. Tutuncuoglu, J. Yang, S. Ulukus, and A. Yener, "Transmis- 973 sion with energy harvesting nodes in fading wireless channels: Optimal 974 policies," IEEE J. Sel. Areas Commun., vol. 29, no. 8, pp. 1732-1743, 975 Sep. 2011.

[3] J. Yang and S. Ulukus, "Optimal packet scheduling in an energy har- 977 vesting communication system," IEEE Trans. Commun., vol. 60, no. 8, 978 pp. 220-230, Jan. 2012.

[4] T. Zhang et al., "A cross-layer perspective on energy harvesting aided 980 green communications over fading channels," IEEE Trans. Veh. Technol., 981 to be published.

[5] C. Huang, R. Zhang, and S. Cui, "Outage minimization in fading channels 983 under energy harvesting constraints," in Proc. IEEE ICC, Jun. 2012, 984 pp. 5788-5793.

985

[6] S. Luo, R. Zhang, and T. J. Lim, "Optimal save-then-transmit protocol for 986 energy harvesting wireless transmitters," IEEE Trans. Wireless Commun., 987 vol. 12, no. 3, pp. 1196-1207, Mar. 2013.

[7] N. Zlatanov, R. Schober, and Z. Hadzi-Velkov, "Asymptotically optimal 989 power allocation for energy harvesting communication networks," IEEE 990 Trans. Inf. Theory, submitted for publication.

[8] C. Huang, R. Zhang, and S. Cui, "Optimal power allocation for outage 992 probability minimization in fading channels with energy harvesting con- 993 straints," IEEE Trans. Wireless Commun., vol. 13, no. 2, pp. 1074-1087, 994 Feb. 2014.

[9] Y. Mao, G. Yu, and Z. Zhang, "On the optimal transmission policy in 996 hybrid energy supply wireless communication systems," IEEE Trans. 997 Wireless Commun., vol. 13, no. 11, pp. 6422-6430, Nov. 2014.

[10] P. He, L. Zhao, S. Zhou, and Z. Niu, "Recursive waterfilling for wireless 999 links with energy harvesting transmitters," IEEE Trans. Veh. Technol., 1000 vol. 63, no. 3, pp. 1232-1241, Mar. 2014.

[11] C. K. Ho and R. Zhang, "Optimal energy allocation for wireless communi- 1002 cations with energy harvesting constraints," IEEE Trans. Signal Process., 1003 vol. 60 , no. 9 , pp. $4808-4818$, Sep. 2012. 
1005 [12] J. Yang and S. Ulukus, "Optimal packet scheduling in a multiple access 1006 channel with energy harvesting transmitters," J. Commun. Netw., vol. 14, 1007 no. 2, pp. 140-150, Apr. 2012.

1008 [13] F. Iannello, O. Simeone, and U. Spagnolini, "Medium access control 1009 protocols for wireless sensor networks with energy harvesting," IEEE 1010 Trans. Commun., vol. 60, no. 5, pp. 1381-1389, May 2012.

1011 [14] H. Li, C. Huang, S. Cui, and J. Zhang, "Distributed opportunistic schedul1012 ing for wireless networks powered by renewable energy sources," in Proc. 1013 IEEE Infocom, 2014, pp. 1-9.

AQ7 1014 [15] Z. Wang, V. Aggarwal, and X. Wang, "Iterative dynamic water-filling 1015 for fading multiple-access channels with energy harvesting," pp. 1-34, 1016 Jan. 2014.

1017 [16] D. Tse and P. Viswanath, Fundamentals of Wireless Communication. 1018 Cambridge, U.K.: Cambridge Univ. Press, 2005.

1019 [17] R. Gallager, "Discrete Stochastic Processes," MIT OpenCourseWare: 1020 Massachusetts Inst. Technol., Cambridge, MA, USA, 2011

1021 [18] I. Krikidis, S. Timotheou, and S. Sasaki, "RF energy transfer for coopera1022 tive networks: Data relaying or energy harvesting?," IEEE Commun. Lett., 1023 vol. 16, no. 11, pp. 1772-1775, Nov. 2012.

1024 [19] V. Kulkarni, A. Forster, and G. Venayagamoorthy, "Computational intel1025 ligence in wireless sensor networks: A survey," IEEE Commun. Surveys 1026 Tuts., vol. 13, no. 99, pp. 1-29, 1st Quart. 2011.

1027 [20] B. Zhang, J. Hu, Y. Huang, M. El-Hajjar, and L. Hanzo, "Outage analysis 1028 of superposition modulation aided network coded cooperation in the pres1029 ence of network coding noise," IEEE Trans. Veh. Technol., vol. 64, no. 2, 1030 pp. 493-501, Feb. 2014

AQ8 1031 [21] B. Zhang, C. Dong, J. Lei, M. El-hajjar, L.-l. Yang, and L. Hanzo, "Buffer1032 aided relaying for the multi-user uplink: Outage analysis and power allo1033 cation," IEEE Trans. Veh. Technol., submitted for publication.

1034 [22] C. Intanagonwiwat, R. Govindan, D. Estrin, J. Heidemann, and F. Silva, 1035 "Directed diffusion for wireless sensor networking," IEEE/ACM Trans. 1036 Netw., vol. 11, no. 1, pp. 2-16, Feb. 2003.

1037 [23] Y. Hou, H. Sherali, and S. Midkiff, "On energy provisioning and relay 1038 node placement for wireless sensor networks," IEEE Trans. Wireless 1039 Commun., vol. 4, no. 5, pp. 2579-2590, Sep. 2005.

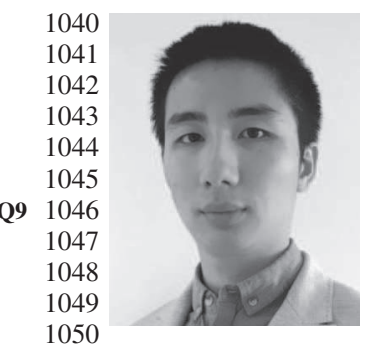

Bo Zhang received the B.Eng. degree in information engineering from the National University of Defense Technology, Changsha, China, in 2010 and the Ph.D. degree in wireless communications from the University of Southampton, Southampton, U.K., in 2015.

$\mathrm{He}$ is currently with the National University of Defense Technology. His research interests in wireless communications include the design and analysis of cooperative communications, multipleinput-multiple-output systems, and network-coded systems.

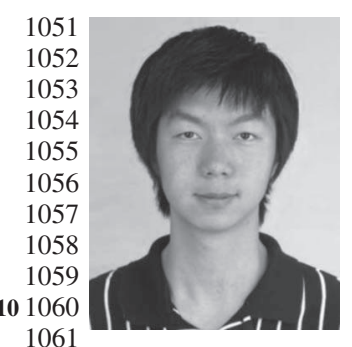

Chen Dong received the B.S. degree in electronic information sciences and technology from the University of Science and Technology of China, Hefei, China in 2004; the M.Eng. degree in pattern recognition and automatic equipment from the University of Chinese Academy of Sciences, Beijing, China, in 2007; and the Ph.D. degree from the University of Southampton, Southampton, U.K., in 2014.

$\mathrm{He}$ is a Postdoctoral Researcher with the University of Southampton His research interests include applied mathematics, relay systems, channel model1062 ing, and cross-layer optimization.

1063 Dr. Dong received a scholarship under the U.K.-China Scholarships for 1064 Excellence Program, and he was awarded the Best Paper Award at the Fall 10652014 IEEE Vehicular Technology Conference.

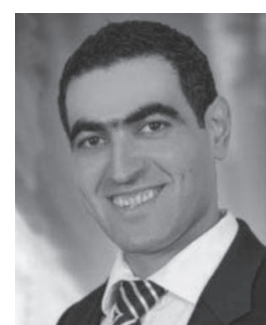

Mohammed El-Hajjar received the B.Eng. degree 1066 in electrical engineering from the American Uni- 1067 versity of Beirut, Beirut, Lebanon, in 2004 and the 1068 M.Sc. degree in radio frequency communication sys- 1069 tems and the Ph.D. degree in wireless communi- 1070 cations, both from the University of Southampton, 1071 Southampton, U.K., in 2005 and 2008, respectively. 1072

After his Ph.D. studies, he joined Imagination 1073 Technologies as a Design Engineer, where he worked 1074 on designing and developing Imagination's multi- 1075 standard communications platform, which resulted 1076 in three patents. In January 2012, he joined the School of Electronics and 1077 Computer Science, University of Southampton, where he is a Lecturer in 1078 the Southampton Wireless Research Group. He has published a Wiley-IEEE 1079 book and more than 50 journal and international conference papers. His 1080 research interests are mainly on the development of intelligent communications 1081 systems, including energy-efficient transceiver design, cross-layer optimization 1082 for large-scale networks, multiple-input-multiple-output systems, millimeter- 1083 wave communications, and radio-over-fiber systems.

1084

Dr. El-Hajjar has received several academic awards, including the Dean's 1085 Award for Creative Achievement, the Dorothy Hodgkin Postgraduate Award, 1086 and the 2010 IEEE International Conference on Communications Best Paper 1087 Award.

1088

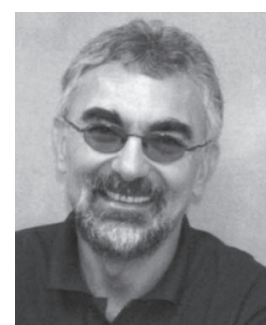

Lajos Hanzo (M'91-SM'92-F'04) received the 1089 Master's degree in electronics, the Ph.D. degree, and 1090 the Doctor Honoris Causa degree from the Technical 1091 University of Budapest, Budapest, Hungary, in 1976, 1092 1983, and 2009, respectively, and the D.Sc. degree 1093 from the University of Southampton, Southampton, 1094 U.K., in 2004.

1095

During his career in telecommunications, he 1096 has held various research and academic posts in 1097 Hungary, Germany, and the U.K. Since 1986, he has 1098 been with the School of Electronics and Computer 1099 Science, University of Southampton, Southampton, U.K., where he holds the 1100 Chair in telecommunications. He was a Chaired Professor with Tsinghua 1101 University, Beijing, China. He is the coauthor of 20 John Wiley/IEEE Press 1102 books on mobile radio communications, totalling in excess of 10000 pages, and 1103 has published more than 1400 research entries on IEEE Xplore. He is currently 1104 directing an academic research team, working on a range of research projects 1105 in the field of wireless multimedia communications sponsored by industry, 1106 the Engineering and Physical Sciences Research Council (EPSRC) U.K., the 1107 European IST Program, and the Mobile Virtual Centre of Excellence, U.K. He 1108 is an enthusiastic supporter of industrial and academic liaison, and he offers a 1109 wide range of industrial courses.

1110

Dr. Hanzo has acted as a Technical Program Committee Chair for IEEE con- 1111 ferences, presented keynote lectures, and has received a number of distinctions. 1112 $\mathrm{He}$ is the Governor of the IEEE Vehicular Technology Society and the Past 1113 Editor-in-Chief of the IEEE Press. 


\section{AUTHOR QUERIES}

\section{AUTHOR PLEASE ANSWER ALL QUERIES}

$\mathrm{AQ1}=$ Please check if the expanded form of RC-UK is properly captured.

AQ2 = Please check if "EH-P2P" is properly captured as "P2P-EH" to maintain consistency in the text.

AQ3 = Please check if "block Rayleigh fading channel" is properly changed to "Rayleigh block-fading channel" to maintain consistency in the text.

AQ4 = Please check if the expanded form of RN is properly captured.

AQ5 = Please provide publication update in ref. [4].

AQ6 = Please provide publication update in ref. [7].

AQ7 = Please provide title of the publication in Ref. [15].

AQ8 = Please provide publication update in ref. [21].

AQ9 = Please check if this sentence is properly inserted to maintain consistency with the current affiliation; otherwise, kindly provide changes.

AQ10 = Please check if the expanded form of FREng, FIET, and EURASIP and VTS and TPC are properly captured.

\section{END OF ALL QUERIES}




\title{
Outage Analysis and Optimization in Single- and Multiuser Wireless Energy Harvesting Networks
}

\author{
Bo Zhang, Chen Dong, Mohammed El-Hajjar, and Lajos Hanzo
}

\begin{abstract}
4 Abstract-Compared to battery-powered wireless nodes having 5 a constant but limited power supply, wireless nodes having energy 6 harvesting (EH) capability may greatly prolong the network's 7 sustainability. However, the energy usage policies (EUPs) have 8 to be carefully designed according to the characteristics of the 9 random power supply gleaned from the environment. In this 10 paper, we carry out the outage analysis of a point-to-point (P2P) 11 network relying on an EH transmitter, which has a finite energy 12 buffer (EB) for transmission over a fading channel when having 13 random energy arrival rates. A discrete Markov chain (DMC) 14 model is proposed for characterizing the energy state of the EB, 15 which is then used for quantifying the outage probability (OP) 16 over the fading channels. Then, we propose both a novel 2-D and a 17 low-complexity 1-D search algorithm for finding the specific EUPs, 18 which are capable of minimizing the OP for the P2P network 19 considered. It is shown that the EUP found by both algorithms 20 outperforms the state-of-the-art EUPs disseminated in the open 21 literature. Furthermore, we consider a multiple-access network 22 having $\boldsymbol{M}$ EH-aided sources, where we propose a distributed 23 EUP optimization (DEUPO) algorithm and then minimize the OP 24 relying on the local optimization of each EH-aided source.
\end{abstract}

26 Index Terms-Energy harvesting (EH), Markov chain, outage analysis, outage minimization.

\section{INTRODUCTION}

28 T practical scenarios such as wireless sensor networks 30 (WSNs), it is challenging to replace the nodes; hence, 31 the network's operation is energy constrained, which is of32 ten formulated as having a limited lifetime [1]. One way of 33 circumventing this problem is allowing the nodes to harvest 34 energy from the environment. If a harvested energy source is 35 permanently available, the transceiver can be powered perpetu36 ally, which fundamentally changes the wireless system design 37 compared to the classic energy-constrained design relying on an 38 energy source storing a limited amount of energy in batteries. 39 Furthermore, based on the periodicity and magnitude of the

Manuscript received December 15, 2014; revised February 19, 2015; accepted February 27, 2015. This work was supported by the Research Councils UK (RC-UK) under the auspices of the India-UK Advanced Technology Center (IU-ATC), by the European Union's Concerto Project, by the European Research Council's Advanced Fellow Grant, and by the Royal Society's Wolfson Research Merit Award. The review of this paper was coordinated by Dr. N.-D. Đào.

B. Zhang is with the School of Electronics and Electrical Engineering, National University of Defense Technology, Changsha 410073, China (e-mail: Bo.Zhang.soton@outlook.com).

C. Dong, M. El-Hajjar, and L. Hanzo are with the School of Electronics and Computer Science, University of Southampton, Southampton SO17 1BJ, U.K. (e-mail: cd2g09@ecs.soton.ac.uk; meh@ecs.soton.ac.uk; 1h@ecs.soton.ac.uk).

Color versions of one or more of the figures in this paper are available online at http://ieeexplore.ieee.org.

Digital Object Identifier 10.1109/TVT.2015.2409781 harvested energy, the transceiver may adjust its energy usage 40 policy (EUP) to improve certain network performance metrics, 41 such as the throughput or outage probability (OP). The EUP 42 may be defined as the "The policy determining the transmitting 43 power and the transmission rate, given the availability of the 44 knowledge on the amount of energy in the energy buffer, the 45 channel statistic information (CSI) as well as the noncausal 46 energy harvesting information (EHI) characterizing the energy 47 arrival rate at the transmitter."

In this paper, we investigate both the effects of random 49 energy arrival and of the EUP design on the OP of wireless 50 energy harvesting (EH) networks. Recently, the EUP design of 51 EH networks has become a hot research area. Various schemes 52 have been proposed in the literature [2]-[9] to improve certain 53 performance metrics in a particular network topology, relying 54 on different assumptions of the energy arrival rates, as well 55 as on the knowledge available at the wireless transceivers for 56 optimization.

Under the idealized simplifying assumption of having both 58 noncausal channel-state information (CSI) about the CSI to be 59 encountered in the future and about the $\mathrm{EH}$ information (EHI) 60 characterizing the energy arrival rate at the transmitter, in [2] 61 and [3], ${ }^{1}$ the optimal offline EUPs were designed for point-to- 62 point (P2P) networks using either the throughput maximiza- 63 tion or the file-transfer completion-time minimization as the 64 optimization objective function (OF). Later on, the authors in 65 [10] proposed the recursive geometric waterfilling algorithm for 66 solving the same problem, where more efficient recursive com- 67 putations were used for finding the optimal solutions. In [4], the 68 authors modeled both the uncertainty of the energy arrival rate 69 and that of the data arrival rate, where the transmission rate to be 70 used was determined by minimizing the average data-buffering 71 delay as the OF.

When the instantaneous CSI is not available at the transmit- 73 ter, having an outage is unavoidable for fixed-rate applications, 74 and the resultant OP of a P2P-EH network was investigated in 75 [5]-[9]. The OP analysis and OP optimization techniques may 76 be categorized into two subclasses according to the knowledge 77 of both the energy arrival rates and the mathematical framework 78 that they adopt; specifically, the first category of contributions 79 recommends the employment of time-variant policies [5], [8], 80 [9]. These authors followed the mathematical framework in [2] 81

\footnotetext{
${ }^{1}$ In [2] and [3], the terminology of "transmission policy" was used to represent the policy of using the harvested energy in the energy buffer (EB). However, the transmission policy terminology may be interpreted more widely, such as rate adaptation, multiple-access policy, etc. Therefore, to avoid ambiguity, we use the terminology of "EUP" throughout the paper.
} 
82 and [11], which adopted the directional waterfilling algorithms 83 under EH-causality constraints ${ }^{2}$ for offline EUP design com84 plemented by the stochastic dynamic programming in online 85 EUP design. The time-variant policy implies the fact that the 86 energy usage would be adapted by relying on the idealized 87 simplifying assumptions of having the a priori knowledge of 88 the instantaneous energy arrival rates. The second category of 89 EUPs recommends time-invariant policies for the long trans90 mission durations routinely encountered in WSNs, which ex91 hibit low computational complexities [6], [7]. The terminology 92 of a time-invariant policy reflects the fact that it does not rely 93 on the idealized knowledge of the instantaneous energy arrival 94 rate, regardless of whether the energy dispensation is designed 95 according to the statistical information of the energy arrival [7] 96 or not [6]. In this case, the EUP may be defined as the "The 97 policy determining the transmitting power, given the amount 98 of energy in the energy buffer and the statistical information 99 of the channel model." Against this backcloth, in this treatise, 100 we aim for filling the gap between the high-complexity time101 variant EUPs and the low-complexity state-of-the-art time102 invariant policies, by considering scenarios having a practical 103 finite EB. As we will show in this paper that the EUPs in the 104 literature [6], [7] did not exploit the EB's state and achieved 105 a suboptimal OP performance. Hence, we propose a range 106 of meritorious methods for improving the OP performance, 107 which fall into the time-invariant category to impose a low 108 computation complexity by relying merely on the knowledge 109 of the average energy arrival rate.

110 As an evolution of research in the subject area of P2P$111 \mathrm{EH}$ networks, the recent contributions on EH strategy design 112 also cover multiple-access EH networks [6], [12]-[15]. In [12], 113 Yang and Ulukus investigated the optimal packet scheduling 114 problem in the context of a two-user fading multiple-access 115 channel. In [15], Wang et al. developed optimal energy schedul116 ing algorithms for a generalized $M$-user fading multiple-access 117 channel relying on $\mathrm{EH}$, to maximize their OF constituted by 118 the network's sum rate, stipulating the idealized simplifying 119 assumption that the side information of both channel states 120 and EH states are known for a certain number of time slots 121 (TSs), where both the battery capacity and the maximum energy 122 consumption during each TS are finite. To the best of our 123 knowledge, the $O P$ minimization problem of a generalized $124 M$-user fading multiple-access channel is, however, an open 125 problem. Against this background, the novel contributions of 126 this paper are as follows.

1) An analytical framework based on a discrete Markov chain (DMC) is proposed for modeling the EB status, for the sake of investigating the OP of a P2P-EH network, in which an EH source node (EH-SN) equipped with a finite EB transmits to a destination node (DN). Given the EB's

\footnotetext{
${ }^{2}$ The EH-causality constraint refers to the fact that, at any time, the transceivers can only utilize the energy that was harvested during the past and the energy not harvested as yet is hence unavailable for usage. Taking into account the causality constraints imposed on the energy usage, the energy can only be saved and used in the future. Therefore, the waterfilling algorithm is redesigned as a directional one, which allows the energy flow only to take place from the past to the future.
}

size and assuming a certain probability distribution func- 132 tion (PDF) for the energy arrival rate, the OP is derived for 133 arbitrary EUPs.

2) We investigate the optimal EUP conceived for minimizing 135 the OP of a P2P-EH network. Based on our proposed ana- 136 lytical framework, we show that constructing an exhaus- 137 tive search for finding the optimal EUP for minimizing 138 the OP is impractical, owing to its excessive complexity, 139 because it scales with $\left(L_{\max }\right)$ !, where $L_{\max }$ is the number 140 of states in the DMC. Therefore, a heuristic 2-D search 141 (2D-search) algorithm is proposed for finding a meritori- 142 ous EUP; we demonstrate that the proposed algorithm is 143 potentially capable of finding the EUP at a manageable 144 complexity. ${ }^{3}$

3) Nonetheless, the 2D-search algorithm conceived still ex- 146 hibits a high complexity; hence, we also propose a low- 147 complexity 1-D search (1D-search) algorithm. We will 148 demonstrate that the OP of the 1D-search algorithm is 149 close to that of its 2D-search counterpart, which may be 150 attractive for applications relying on low-cost hardware, 151 such as mobile phones and wireless sensors.

4) We extend the proposed DMC framework to more general 153 nonorthogonal EH networks. In contrast to the P2P sce- 154 nario, the outage events of practical EH-SNs tend to be 155 correlated. As an attractive application scenario, we will 156 investigate the OP of maximum-likelihood (ML) detection 157 in the context of spatial-division multiple-access (SDMA) 158 networks, we will decompose the OP by approximat- 159 ing it as multiple independent outage probabilities, each 160 corresponding to a simple P2P-EH-network subproblem. 161 Finally, we will propose a distributed EUP optimization 162 (DEUPO) protocol, where each EH-SN is capable of 163 optimizing its own policy using both the local statistics 164 of the fading channel and the related energy arrival model. 165

The rest of this paper is organized as follows: In Section II, 166 we first discuss the EUPs found in the literature and then invoke 167 the DMC for modeling the EB's state. Based on this model, 168 we consider the OP minimization problem and propose the 169 aforementioned 2D-search and 1D-search algorithms conceived 170 for finding the optimal EUPs. In Section III, we investigate 171 the EUP design of SDMA-EH networks, and we propose the 172 aforementioned distributed DEUPO protocol. Finally, our con- 173 clusions are presented in Section V.

\section{Peer-To-Peer-Energy Harvesting Network 175} DESIGN

\section{A. System Model and OP Formulation}

We first consider a simple P2P network constituted by an 179 SN and a DN, which is shown in Fig. 1. As shown in Fig. 1, 180

\footnotetext{
${ }^{3}$ When the Markov chain model has $L_{\max } \leq 10$ states and the number of OF evaluations is lower than 10!, the exhaustive searching may be implemented and therefore may serve as the benchmark for our proposed algorithm. However, for $L_{\max }>10$, the complexity becomes excessive, which prevents us from verifying, whether the $2 \mathrm{D}$-search algorithm is capable of matching the optimal EUP. On the other hand, it is challenging to mathematically prove the optimality of a search algorithm in the context of a nonconvex problem involving highdimensional matrices. Therefore, this open problem will be further detailed in our discussions, and it will be investigated in our future work.
} 


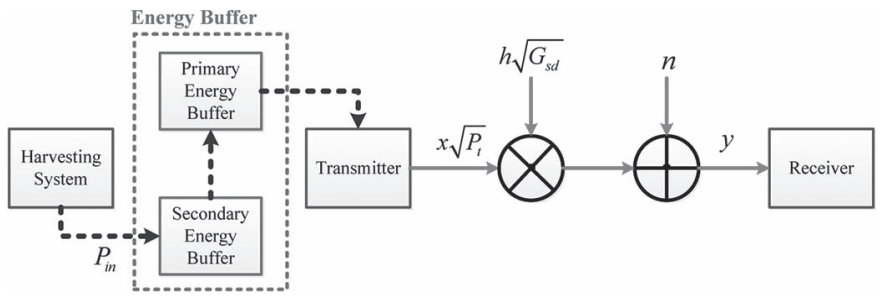

Fig. 1. System model of the P2P-EH network.

181 a primary $\mathrm{EB}$ and a secondary $\mathrm{EB}$ is required in practice 182 [1], [6]. In [1], the secondary storage is a backup storage 183 invoked for situations, when the primary storage is exhausted. 184 In [6], the authors assumed that the rechargeable energy storage 185 devices cannot charge and discharge simultaneously; hence, the 186 transmitter is powered by the primary EB for data transmission, 187 while the secondary EB is connected to the harvesting system 188 and charges up. At the end of the recharge cycle, the secondary 189 EB would be charged by the secondary EB. We assume that 190 the charging time of the primary EB is negligible ${ }^{4}$ and that the 191 charging efficiency is assumed to be $100 \% .^{5}$ Therefore, both 192 the primary and the secondary EBs may be represented by a 193 single EB, which is represented by the dashed-line box shown 194 in Fig. 1. This buffer is assumed to be capable of powering the 195 transmitter, while simultaneously being charged by the harvest196 ing system. We do not make any specific assumptions as to what 197 harvesting system is adopted, which may be solar cells, a wind 198 anemometer, etc., as discussed in [1]. We assume that the EB at 199 the SN has a finite EB size, where the harvested energy is stored 200 and used for transmission. We assume furthermore that the 201 energy arrival rate $P_{\text {in }}$ obeys a certain probability distribution 202 with an expectation of $\bar{P}_{\text {in }}$, and it remains constant over a TS of 203 duration $T_{E}$, while changing independently over the subsequent 204 TSs, where a time slot is a recharge cycle. We assume that 205 the instantaneous energy arrival rate is unknown and cannot be 206 used during the current TS of $T_{E}$, because the secondary EB is 207 not allowed to charge and discharge simultaneously, as shown 208 in Fig. 1. In order to focus our attention on the EUP conceived 209 for wireless transmission, we assume that the circuit power con210 sumption at the $\mathrm{SN}$ is negligible and that the energy conversion 211 efficiency between the EB and the transmit power is $100 \%{ }^{6}$

212 Let us now consider the channel modeling of the wireless 213 communication links. We consider a narrow-band block-fading 214 channel model, where the fading coefficients remain constant 215 for the duration of a transmission packet denoted by $T_{C}$ and 216 then they are faded independently from one packet to another

\footnotetext{
${ }^{4}$ In practice, this may be realized by a supercapacitor-based storage system, such as, for example, the Everlast solar system introduced in [1].

${ }^{5}$ In practice, the charging efficiency of the secondary EB may not reach $100 \%$; hence, it may be multiplied by an efficiency factor $\eta_{\text {buffer }} \in[0,1]$, which may be equivalently considered to be a reduced energy arrival rate, and hence, it does not affect any of our analysis.

${ }^{6}$ In practice, the power consumption of the circuits may be nonnegligible. We may assume that the harvesting system is capable of providing sufficient circuit power, while additionally providing a nonnegative transmit power. When the $\mathrm{EH}$ system is not capable of supplying sufficient circuit power, the transmitter may be switched off. On the other hand, the energy conversion efficiency $\eta_{\mathrm{TX}}$ from the EB to the transmitter cannot reach $100 \%$ in practice. Hence, we may simply multiply the energy arrival rate at the transmitter with an efficiency coefficient $\eta_{\mathrm{TX}} \in[0,1]$, which does not affect any of our analysis.
}

over the time dimension. Note that we make no assumptions 217 concerning the specific channel model and the distribution of 218 the channel gain. We also assume that there are always data 219 packets buffered at the $\mathrm{SN}$ for transmission. The signal received 220 at the DN is represented by

$$
y=h \sqrt{P_{t}} G_{s d} x+n
$$

where $h$ is the channel coefficient capturing the effects of fad- 222 ing, while $P_{t}$ is the transmit power, $x$ is the transmitted signal, 223 and $n$ is the additive noise at the receiver, which is modeled by 224 independent standard circularly symmetric complex Gaussian 225 random variables having a zero mean and a variance of 1 . In (1), 226 the average processing gain of $G_{s d}=\left(N_{0} \times d_{s d}^{\beta}\right)^{-1}$ between 227 the $\mathrm{SN}$ and the DN captures the effect of both the pathloss 228 and the noise, where $N_{0}$ is the noise power at the receiver, $d_{s d}$ is 229 the distance between the $\mathrm{SN}$ and the DN, while $\beta$ is the pathloss 230 exponent.

An outage is defined as the event when the instantaneous 232 received signal-to-noise power ratio (SNR) $\gamma$ at the receiver 233 is below a predefined threshold $\gamma_{\text {th }}$ that has to be exceeded 234 for successful decoding. If idealized perfect capacity-achieving 235 coding is assumed, we have $\gamma_{\text {th }}=2^{R}-1$, where $R$ is the 236 data transmission rate [16]. Then, the OP of the single-hop EH 237 network may be expressed as follows:

$$
\begin{aligned}
P_{\text {out }} & =\operatorname{Pr}\left\{P_{t}|h|^{2} G_{s d}<\gamma_{\mathrm{th}}\right\} \\
& \triangleq \operatorname{Pr}\left\{P_{t}|h|^{2}<P_{\mathrm{th}}\right\}
\end{aligned}
$$

where $P_{t}$ is the transmit power, and $h$ is the normalized channel 239 coefficient capturing the fading effects. In (2), we define $P_{\text {th }}=240$ $\gamma_{\mathrm{th}} / G_{s d}$, to focus our attention on the effects of both the 241 transmit power $P_{t}$ and the channel's fading coefficient $h . \quad 242$

In the conventional transmission scheme relying on classic 243 constant power supply, the transmit power $P_{t}$ is a constant, and 244 the corresponding OP of narrow-band block-fading channels 245 was quantified in [16]. However, in the EH networks, the instan- 246 taneous transmit power $P_{t}$ is time variant, which is constrained 247 by the amount of the energy available in the EB, which in turn 248 is a random variable depending on the energy arrival rate. The 249 energy arrival rate is assumed to exhibit a blockwise fluctuating 250 nature, which remains constant over a TS of duration $T_{E}$ and 251 changes independently over the subsequent TSs. During a TS 252 with a duration of $T_{E}$, the amount of energy harvested, i.e., 253 $P_{\text {in }} T_{E}$, is independent of both that harvested in the previous TS 254 and of the energy consumed, i.e., $P_{t} T_{E}$, during transmission, 255 which is determined by the EB state $B_{T}$ at the beginning of the 256 current TS.

We define the EB state as $B_{T}=B_{E} / T_{E}$, where $B_{E}$ is the 258 amount of energy available in the EB, while $T_{E}$ is the duration 259 of the recharge cycle. The physical interpretation of $B_{T}$ is the 260 maximum average transmit power that may be supported by 261 the amount of energy stored in the buffer during the current 262 recharge cycle. ${ }^{7}$ The EH-causality constraint [2] is interpreted 263

\footnotetext{
${ }^{7}$ When the knowledge of the instantaneous CSI during a period is unavailable at the transmitter, transmitting at a constant transmit power would achieve the minimum OP [16]. Therefore, a constant transmit power is adopted during each recharge cycle, and $B_{T}$ is the upper bound.
} 


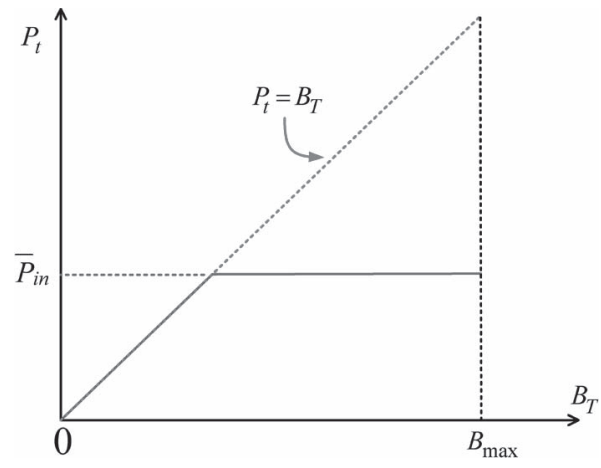

Fig. 2. EUP illustrated as the function of $P_{t}$ versus $B_{T}$.

264 as follows: the instantaneous transmit power $P_{t}$ cannot exceed 265 the maximum power $B_{T}$ that may be supported by the current $266 E B$ state, i.e., we have $P_{t} \leq B_{T}$, explicitly indicating that the 267 energy assigned for transmission must not exceed the amount 268 of energy harvested. We may model the EUP by the transmit 269 power as a function of the EB state, as follows:

$$
P_{t}\left(B_{t}\right), B_{T} \in\left[0, B_{\max }\right]
$$

270 where the EB state $B_{T}$ is upper bound by $B_{\max }$ defined as the 271 EB capacity divided by the recharge cycle $T_{E}$.

272 In Fig. 2, the EH-causality constraint is shown in dashed 273 lines as $P_{t}\left(B_{T}\right)=B_{T}$, which models the best-effort policy 274 proposed in [6], where all harvested energy in the buffer is 275 used up for transmission. On the other hand, the asymptotic 276 optimal policy proposed in [7] is illustrated by the solid line in 277 Fig. 2, where the SN aims to transmit at a power of $P_{t}=\bar{P}_{\text {in }}$. 278 In the asymptotic optimal policy, when the remaining energy in 279 the EB is capable of supporting a higher transmit power than 280 the average energy arrival rate $\bar{P}_{\text {in }}$, the transmitter conserves 281 the energy for its future usage. If the remaining energy in the $282 \mathrm{~EB}$ is insufficient for supporting $P_{t}=\bar{P}_{\text {in }}$, the SN switches to 283 the best-effort policy. We may formulate the OP of the P2P-EH 284 network as follows:

$$
\begin{aligned}
P_{\text {out }}\left(P_{t}\right) & =\int_{0}^{B_{\max }} \operatorname{Pr}\left\{P_{t}(x)|h|^{2}<P_{\mathrm{th}}\right\} f_{B_{T}}(x) d x \\
& =\int_{0}^{B_{\max }} \int_{0}^{+\infty} \operatorname{Pr}\left\{P_{t}(x) y<P_{\mathrm{th}}\right\} f_{|h|^{2}}(y) f_{B_{T}}(x) d x
\end{aligned}
$$

285 where $h$ is the channel coefficient capturing the effects of 286 fading, which is a random variable, and its PDF $f_{|h|^{2}}(y)$ relies 287 on the statistical channel model. $f_{B_{T}}(x), x \in\left[0, B_{\max }\right]$ is the $288 \mathrm{PDF}$ of the EB state $B_{T}$. Therefore, to derive the OP formulated 289 in (4), the PDF of the EB state $B_{T}$ has to be modeled, bearing in 290 mind the specific EUP adopted. Furthermore, because both $P_{t}$ 291 and $B_{T}$ are continuous variables, the number of feasible EUPs 292 is infinite, and since different policies would result in different 293 EB-state PDFs, finding the optimal policy for minimizing the 294 OP in (4) may be quite challenging. Hence, we will investigate 295 this problem in the next section.
As the energy arrival rate $P_{\text {in }}$ is assumed to be constant over 297 a recharge cycle $T_{E}$ and then changes independently over the 298 subsequent recharge cycles, the EB state $B_{T}(k)$ at the end 299 of the $k$ th $(k \geq 1)$ recharge cycle relies only on the state of 300 $B_{T}(k-1)$, on the amount of energy consumed for transmis- 301 sion $P_{t}\left[B_{T}(k)\right]$, as well as on the current energy arrival rate $P_{\mathrm{in}}, 302$ which obeys a certain PDF, but it is statistically independent 303 of its previous samples. Therefore, $B_{T}$ may be modeled by a 304 continuous Markov process.

However, the domain of $B_{T} \in\left[0, B_{\max }\right]$ is continuous; 306 hence, the set of the states is uncountable and challenging to 307 manage [17]. Therefore, given the EUP, deriving the PDF of $B_{T} 308$ is quite challenging, except for certain special cases, such as the 309 best-effort policy combined with the condition, when the trans- 310 mit power is equal to the instantaneous arriving energy, which 311 may be modeled by the exponential distribution [6]. Even for 312 the asymptotic optimal policy [7], where $P_{t}$ is a simple function 313 determined by a combination of the best-effort policy and of the 314 constant power supply, the PDF of $B_{T}$ cannot be readily derived 315 in closed form; hence, the asymptotic optimality relies on the 316 fact that the probability of $\operatorname{Pr}\left\{B_{T}<P_{t}=\bar{P}_{\text {in }}\right\} \rightarrow 0$, when the 317 EB size obeys $B_{\max } \rightarrow \infty$. In order to quantify and then to 318 minimize the OP in (4), we approximate the continuous-state 319 Markov process by a finite-state Markov chain [18], to model 320 the EB state $B_{T}$, and to derive the PDF of $B_{T}$. Specifically, the 321 EB size $B_{\max }$ is discretized as $L_{\max }=\left\lfloor B_{\max } / \varepsilon_{P}\right\rfloor$, where $\varepsilon_{P} 322$ is the discrete step size of the power. Therefore, $l=\left\lfloor B_{T} / \varepsilon_{P}\right\rfloor 323$ may take a value from $l \in\left\{0,1, \ldots, L_{\max }\right\}$ and has a state- 324 space size of $\left(L_{\max }+1\right)$. The instantaneous EH rate $P_{\text {in }}$ and 325 the decoding threshold $P_{\text {th }}$ are also discretized with a step size 326 of $\varepsilon_{P}$ as

$$
\begin{aligned}
L_{\text {in }} & =\left\lfloor\frac{P_{\text {in }}}{\varepsilon_{P}}\right\rfloor \\
L_{\text {th }} & =\left\lfloor\frac{P_{\mathrm{th}}}{\varepsilon_{P}}\right\rfloor .
\end{aligned}
$$

Hence, $L_{\mathrm{th}}$ is a discrete constant when $P_{\mathrm{th}}$ is given, while $l$ and 328 $L_{\text {in }}$ are discrete random variables, and their probability mass 329 functions (PMFs) may be generated from the PDFs of $B_{T}$ and 330 $P_{\text {in }}$ as follows:

$$
\begin{aligned}
\operatorname{Pr}\{l=x\} & =\int_{x \varepsilon_{P}}^{(x+1) \varepsilon_{P}} f_{B_{T}}(u) d u \\
\operatorname{Pr}\left\{L_{\text {in }}=x\right\} & =\int_{x \varepsilon_{P}}^{(x+1) \varepsilon_{P}} f_{P_{\text {in }}}(u) d u .
\end{aligned}
$$

Although the variables $B_{T}, P_{\mathrm{in}}$, and $P_{t}$ may assume any arbi- 332 trary continuous nonnegative value, the DMC may be capable 333 of sufficiently accurately capturing the buffer's behavior, as 334 long as the discretization step size $\varepsilon_{P}$ is small enough. Finally, 335 we may discretize the EUP formulated in (3) as

$$
P_{t}(l)=P_{t}\left(\left\lfloor\frac{B_{T}}{\varepsilon_{P}}\right\rfloor\right), l \in\left\{0,1, \ldots, L_{\max }\right\}
$$


337 where the discrete EUP is defined as

$$
L_{t}(l)=\left\lfloor\frac{P_{t}(l)}{\varepsilon_{P}}\right\rfloor .
$$

338 Then, we may construct the state transition matrix $T$ of the EB 339 states, where the specific element in the $i$ th row and $j$ th column 340 is given by

$$
\begin{aligned}
T_{i, j} & =\operatorname{Pr}\{l(k+1)=j \mid l(k)=i\} \\
& = \begin{cases}\operatorname{Pr}\left\{j=i+L_{\text {in }}-L_{t}(i)\right\}, & 0 \leq j<L_{\text {max }} \\
\operatorname{Pr}\left\{j \leq i+L_{\text {in }}-L_{t}(i)\right\}, & j=L_{\text {max }} .\end{cases}
\end{aligned}
$$

341 We arrive at the steady-state probability vector $\pi=\left[\begin{array}{ll}\pi_{0} & \pi_{1}\end{array} \ldots\right.$ $\left.342 \pi_{L_{\max }}\right]^{T}$ using the relationship of

$$
\pi=T^{T} \pi
$$

343 where the physical interpretation of (10) is that the state proba344 bility vector $\pi$ converges and remains constant. Then, we may 345 formulate the OP as

$$
\begin{aligned}
P_{\text {out }}\left(L_{t}(l)\right) & =\sum_{l=0}^{L_{\text {max }}} \operatorname{Pr}\left\{L_{t}(l)|h|^{2}<L_{\mathrm{th}}\right\} \pi(l) \\
& \triangleq \sum_{l=0}^{L_{\max }} P_{e}(l) \pi(l)
\end{aligned}
$$

346 which is the discrete version of (4). It should be noted that, 347 in (11), the OP component of $P_{e}(l) \triangleq \operatorname{Pr}\left\{L_{t}(l)|h|^{2}<L_{\mathrm{th}}\right\}$ is 348 not determined unambiguously by the EUP defined by $L_{t}(l)$, $349 l \in\left[0, L_{\max }\right]$, because it also relies on the statistical channel 350 model determining the distribution of $|h|^{2}$. For example, if a 351 narrow-band Rayleigh block-fading channel is assumed, then $352|h|^{2}$ follows the exponential distribution in conjunction with the 353 parameter of 1 . In this case, the OP component $P_{e}(l)$ may be 354 expressed as

$$
P_{e}(l)=\operatorname{Pr}\left\{L_{t}(l)|h|^{2}<L_{\mathrm{th}}\right\}=1-e^{-\frac{L_{\mathrm{th}}}{L_{t}(l)}} .
$$

\section{C. Two-Dimensional EUP-Search Algorithm}

356 Given a certain EUP represented by $L_{t}(l), l \in\left[0, L_{\max }\right]$ and 357 a specific statistical channel model, we are now capable of 358 quantifying the OP of a certain EUP with the aid of (7)-(11). 359 The optimal EUP $L_{t}(l), l \in\left[0, L_{\max }\right]$ may be formulated by 360 using the physically meaningful OF minimizing the $\mathrm{OP}$ as 361 follows:

$$
\min _{L_{t}(l)} P_{\text {out }}\left[L_{t}(l)\right]
$$

362 However, the inverse of the mapping in (11) from the OP $363 P_{\text {out }}\left[L_{t}(l)\right]$ to the specific EUP $L_{t}(l)$ cannot be readily evalu364 ated. In other words, given a certain $P_{\text {out }}[L(l)]$, it is not possible 365 to derive the EUP $L_{t}(l)$ adopted. Naturally, this hinders the related inverse mapping, and hence, the closed-form derivation 366 of the optimal EUP is not possible. Although the buffer-state 367 transition matrix $T$ of (9) may be readily determined, given 368 the EUP $L_{t}(l)$, according to (9), the resultant steady-state 369 probability vector $\pi=\left[\begin{array}{lllll}\pi_{0} & \pi_{1} & \ldots & \pi_{L_{\max }}\end{array}\right]^{T}$ is a solution of 370 (10), which is a high-dimensional system of linear equations. 371 Furthermore, given a certain steady-state probability vector $\pi, 372$ it is not possible to derive the buffer-state transition matrix $T, 373$ and hence, we cannot uniquely and unambiguously determine 374 the discrete EUP $L_{t}(l)$.

1) Design Motivations: When using a discrete Markov mod- 376 eling of the EB state, the EUP is represented by a vector of 377 $L_{t}(l), l \in\left[0, L_{\max }\right]$, which has $\left(L_{\max }+1\right)$ legitimate elements 378 over the first dimension constituted by the EB state, where the 379 $l$ th element in $L_{t}(l)$ itself may be assigned any discrete value 380 spanning from 0 to $l$ over the second dimension representing the 381 amount of energy assigned for transmissions. Hence, the EUP 382 search is over a 2-D space. The aforementioned fact motivates 383 us to design an EUP-search algorithm. The most conceptually 384 straightforward way of finding the optimal EUP $L_{t}(l), l \in 385$ $\left[0, L_{\max }\right]$ is to invoke an exhaustive search, which evaluates 386 every feasible EUP and selects the one having the minimum 387 OP. As illustrated in Fig. 2, an EUP $L_{t}(l)$ is physically feasible 388 as long as the instantaneous transmit power $P_{t}$ is nonnegative 389 and does not exceed the maximum affordable power $B_{T}$ that 390 may be supported by the current EB state $P_{t} \leq B_{T}$, which is 391 equivalent to the following discrete form:

$$
0 \leq L_{t}(l) \leq l, \forall l \in\left[0, L_{\max }\right]
$$

This simple feasibility constraint results in a large num- 393 ber of feasible EUPs, where the complexity of searching for 394 the optimal policy that minimizes the OP may be excessive. 395 Quantitatively, there are $N_{f}=\left(L_{\max }+1\right)$ ! number of feasible 396 functions of $L_{t}(l)$, given the condition in (14). For example, if 397 we have $L_{\max }>11$, the number of feasible functions becomes 398 $N_{f}>10^{8}$. Therefore, the exhaustive search method of finding 399 the optimal policy is not practically feasible. Hence, we have 400 to design search algorithms having a practically tolerable com- 401 plexity, which are detailed in the following sections. 402

2) EUP-Search Algorithm Design: In the algorithms pro- 403 posed in this treatise, the design guidelines that we adopted for 404 controlling the complexity, which is quantified by the number 405 of $O P$ evaluations, are summarized as follows.

- Guideline 1: The optimal EUP $L_{t}(l), l \in\left[0, L_{\max }\right]$ is a 407 nondecreasing function of the EB state l, i.e., we have 408 $\forall k \in\left[0, L_{\max }-1\right], L_{t}(k+1)-L_{t}(k) \geq 0$. The physi- 409 cal interpretation of this guideline can be summarized as 410 follows. If the amount of energy available in the EB is 411 increased, the transmitter should not use a lower transmit 412 power. The reason behind this guideline is twofold: First, 413 the transmitter has no knowledge of the energy arrival rate 414 in the future; therefore; it cannot decide as to whether 415 conserving the harvested energy in the EB for future usage 416 is beneficial. Second, the transmitter has no knowledge of 417 the instantaneous channel gain; therefore, it cannot decide 418 how to control the transmit power. 
441 Although the aforementioned pair of design guidelines may 442 be interpreted physically in a simple manner, it is challeng443 ing to rigorously prove the optimality of Guideline 1, while 444 Guideline 2 is applied in a relatively high transmit power 445 scenario associated with a good channel quality, when the OP 446 is a convex function of the transmit power [8]. When relying 447 on the proposed pair of design guidelines, the number of OP 448 evaluations is reduced from $N_{f}=\left(L_{\max }\right)$ ! to $N_{2 D}=2^{N_{\max }}$, 449 which may still be excessive. Quantitatively, when we have $450 N_{\max }>30$, the number of OF evaluations obeys $N_{2 D}>10^{9}$. 451 Therefore, we conceive a third guideline for controlling the 452 complexity, albeit this is achieved at the cost of potentially 453 resulting in a locally optimal solution, which is detailed as 454 follows.

462 Since Guideline 3 may result in locally optimal solutions, 463 multiple initial solutions may be chosen for the search al464 gorithm. However, through our extensive numerical evalua465 tions conducted for $N_{\max }<12$, when the exhaustive search 466 algorithm is still feasible, our numerical results have shown 467 that Algorithm 1 is capable of finding the globally optimal 468 EUP. Algorithm 1 uses the best-effort policy as the initial 469 solution, and then, the three aforementioned guidelines are 470 followed throughout the rest of the design. Therefore, it may 471 be concluded that, although the optimality may not be shown 472 mathematically, the proposed heuristic 2D-search algorithms 473 are effective in practical applications, while imposing a much 474 lower complexity than the exhaustive search.

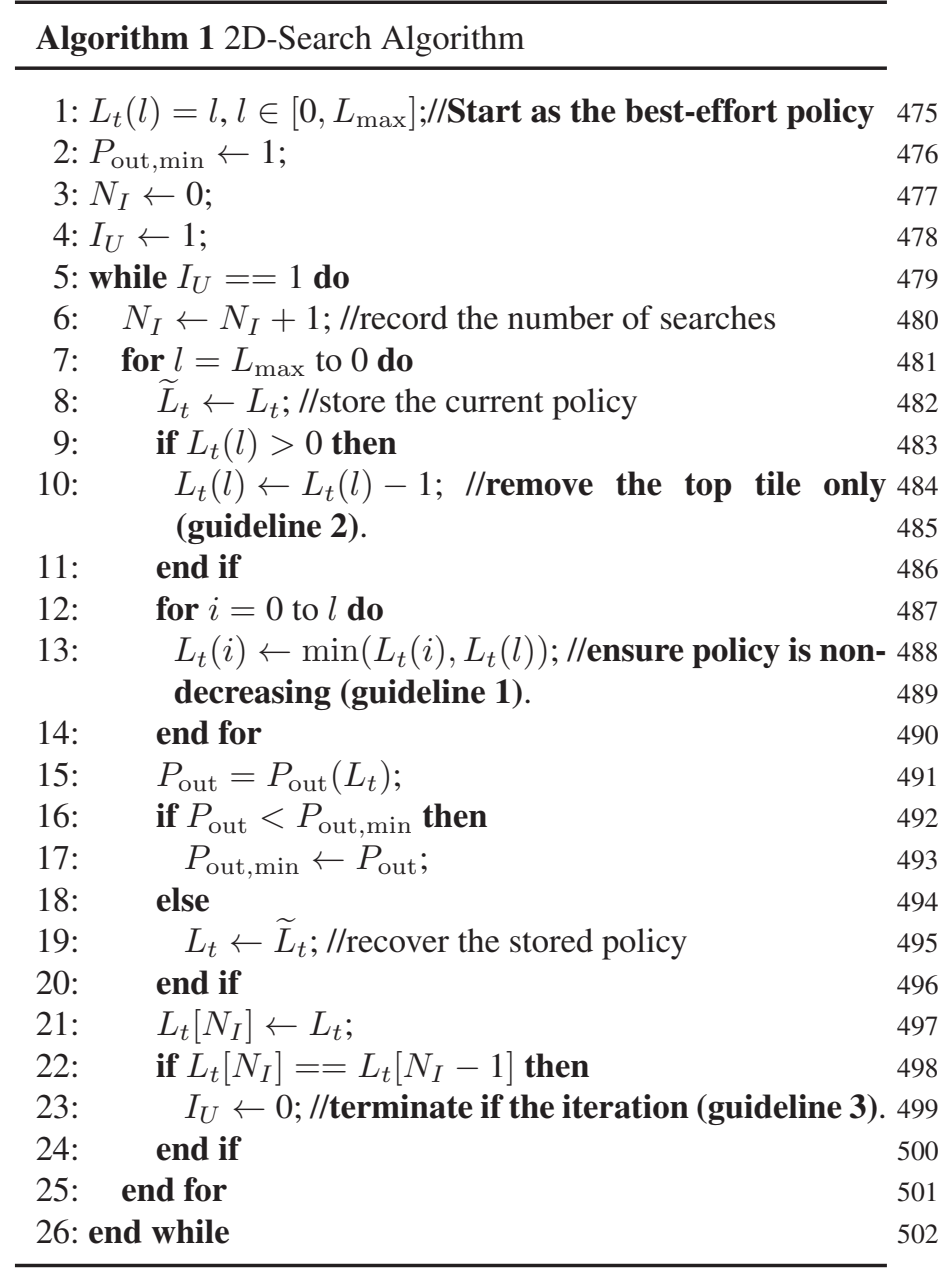

\section{One-Dimensional EUP-Search Algorithm}

In the previous section, the optimal EUP was investigated and 504 a 2D-search algorithm was proposed. However, the algorithm 505 relies on searching in a 2-D domain of the EB state and of the 506 energy assigned for transmission; hence, it is quite involved. 507 Here, motivated by the fact that the asymptotic optimal policy 508 is characterized by a constant desired transmit power [7], we 509 formulate a 1D-search-based EUP and aim for minimizing the 510 OP using a reduced-complexity 1-D search to exhibit a signifi- 511 cantly lower complexity than that of the 2D-search algorithm. 512

1) Design Motivations: Our proposed 1D-search policy is 513 motivated by the asymptotic optimal policy proposed in [7], 514 which is illustrated in Fig. 2. The suboptimal EUP considered 515 is based on a combination of the constant power policy and the 516 best-effort policy. Specifically, given a desired constant transmit 517 power $P_{d}$, when the energy remaining in the EB satisfies 518 $B_{t} \geq P_{d}$, the transmitter opts for transmitting at a power of 519 $P_{t}=P_{d}$ and conserves the rest of the energy for its future 520 usage. Otherwise, when $B_{t}<P_{d}$, the transmitter switches to 521 the best-effort policy and transmits at a power of $P_{t}=B_{T}$. The 522 suboptimal policy is represented by a fixed $P_{t}\left(B_{T}\right)$ of

$$
P_{t}\left(B_{T}\right)= \begin{cases}B_{T}, & B_{T}<P_{d} \\ P_{d}, & B_{T} \geq P_{d}\end{cases}
$$


524 while its discrete version represented by $L_{t}(l), l \in\left[0, L_{\max }\right]$ is

$$
L_{t}(l)= \begin{cases}l, & l<L_{d} \\ L_{d}, & l \geq L_{d}\end{cases}
$$

525 where we define $L_{d}=\left\lfloor P_{d} / \varepsilon_{P}\right\rfloor$. Compared to the generalized 526 representation of $L_{t}(l), l \in\left[0, L_{\max }\right]$, which requires $\left(L_{\max }+\right.$ 527 1) variables for fully characterizing the policy, the proposed 528 EUP may be characterized by a single variable $L_{d}$. Therefore, $529 L_{d}$ is also the only variable that may be optimized to minimize 530 the OP. However, the 1D-search policy may be expected to 531 result in a degraded OP.

532 A special case of the proposed EUP is to set $P_{d}=\bar{P}_{\text {in }}$ or 533 equivalently $L_{d}=\bar{L}_{\text {in }}$. The asymptotic optimal EUP proposed 534 in [7] was shown to achieve the performance of its constant535 power counterpart operating at $P_{t}=\bar{P}_{\text {in }}$, based on the assump536 tion of an infinite EB size of $B_{\max } \rightarrow \infty$ [7]. In this case, 537 the probability of an EB overflow is 0 , and the probability 538 of $\operatorname{Pr}\left\{B_{T}<P_{d}\right\}=\operatorname{Pr}\left\{l<L_{d}\right\} \rightarrow 0$. It is plausible that the 539 performance of the classic non-EH system constitutes the OP 540 lower bound that may be achieved by any EH system relying 541 on a random energy arrival rate. Naturally, achieving the per542 formance of the asymptotic optimal EUP is desirable [7].

543 However, when the EB size is finite, the asymptotic optimal 544 policy would be suboptimal, because a finite EB may overflow 545 with a nonnegligible probability, when the instantaneous energy 546 arrival rate is high and cannot be stored for future usage. 547 Meanwhile, the choice of $L_{d}=\bar{L}_{\text {in }}$ may not be optimal, since 548 a choice of $L_{d} \neq \bar{L}_{\text {in }}$ may reduce both the probability of EB 549 overflow and the OP. However, the optimal choice ${ }^{8}$ of $P_{d}$ is 550 not obvious, because the relationship between the OP $P_{\text {out }}$ and 551 the energy usage function $L_{t}$ is quantified by (9)-(11), which 552 makes the direct derivation of the optimal $P_{d}$ quite challenging. 553 By comparison, as shown in (7)-(11), given a specific value 554 of $P_{d}$, the numerical evaluation of $P_{\text {out }}$ may be straightforward, 555 according to the OP expression provided in (11). This motivates 556 us to design a search algorithm, which searches for the optimal $557 P_{d}$ based on the numerical evaluation of $P_{\text {out }}$, instead of using 558 an analytical derivation to get the optimal $P_{d}$ directly.

559 In the next section, we will first derive the OP for the 1D560 search-based EUP given a specific $L_{d}$ and then propose our 561 specific search algorithm for finding the optimal $L_{d}$ to minimize 562 the OP.

563 2) One-Dimensional EUP-Search Algorithm Design: Upon 564 invoking the 1D-search-based EUP represented in (16), we may 565 simplify the OP expression of (11) specifically for the 1D566 search policy as follows:

$$
\begin{aligned}
P_{\text {out }}=\operatorname{Pr}\{l & \left.\geq L_{d}\right\} \operatorname{Pr}\left\{L_{d}|h|^{2}<L_{\text {th }}\right\} \\
& +\operatorname{Pr}\left\{l<L_{d}\right\} \operatorname{Pr}\left\{l|h|^{2}<L_{\text {th }} \mid l<L_{d}\right\}
\end{aligned}
$$

567 where the first line represents the OP, when the energy in the $568 \mathrm{~EB}$ is capable of supporting transmitting at the desired level of $569 L_{d}$. The second line in (17) represents the OP, when the energy 570 in the EB is insufficient for transmitting at the power level of

\footnotetext{
${ }^{8}$ The optimal choice is in the context of selecting $P_{d}$ for the $1 \mathrm{D}$-search algorithm, which may still result in inferior OP compared to the exhaustive search and the 2D-search algorithms.
}

$L_{t}=L_{d}$, and the transmitter consumes all the energy in the 571 EB, while transmitting at a power level of $L_{t}=l$. Then, we 572 construct the state transition matrix $T$ of the EB state according 573 to (9), and when the EB state is steady, the state probability 574 vector $\pi$ may be formulated as follows:

$$
\pi=T^{T} \pi
$$

where $\pi=\left[\begin{array}{llll}\pi_{0} & \pi_{1} & \ldots & \pi_{L_{\max }}\end{array}\right]^{T}$. Given the desired power level 576 represented by $L_{d}$ and the OP expression in (17), we have 577

$$
\operatorname{Pr}\left\{l \geq L_{d}\right\}=\sum_{l=L_{d}}^{L_{\max }} \pi_{l} .
$$

If we assume furthermore that the channel obeys Rayleigh 578 fading, the other terms in (17) can be derived as follows:

$$
\begin{aligned}
& \operatorname{Pr}\left\{L_{d}|h|^{2}<L_{\mathrm{th}}\right\}=1-\exp \left(-\frac{L_{\mathrm{th}}}{L_{d}}\right) \\
& \operatorname{Pr}\left\{l<L_{d}\right\} \operatorname{Pr}\left\{l|h|^{2}<L_{\mathrm{th}} \mid l<L_{d}\right\} \\
& =\sum_{l=0}^{L_{d}-1} \pi_{l} \operatorname{Pr}\left\{l|h|^{2}<L_{\mathrm{th}}\right\} \\
& =\sum_{l=0}^{L_{d}-1} \pi_{l}\left[1-\exp \left(-\frac{L_{\mathrm{th}}}{l}\right)\right] .
\end{aligned}
$$

By substituting the terms of (18)-(20) into (17), we may arrive 580 at the analytical OP for transmission over Rayleigh block- 581 fading channels in the P2P-EH network in Fig. 1. If a differ- $582 \mathbf{A Q 3}$ ent statistical channel model is adopted, we may reformulate 583 (19) and (20), accordingly. Throughout this paper, we use the 584 Rayleigh block-fading channel as a case study, although our 585 proposed OP analysis and the search algorithms conceived for 586 OP minimization are sufficiently general for arbitrary channel 587 models. The effects of other wireless channel models will be 588 investigated in our future research.

589

Therefore, given a specific value of $L_{d}$, the numerical eval- 590 uation of $P_{\text {out }}$ is straightforward, according to the OP expres- 591 sion provided in (17). Since it relies on the single parameter 592 $L_{d}$, a 1-D EUP-search algorithm may be designed for finding 593 the optimal $L_{d}$, instead of searching over a 2-D EUP space, 594 as in Section II-C. This 1D-search procedure is detailed in 595 Algorithm 2, which is much simpler than the 2D-search algo- 596 rithm in Section II-C. Specifically, in Algorithm 2, there are a 597 total of $\left(L_{\max }+1\right)$ candidate EUPs, since we have $L_{d} \in\{0,1,598$ $\left.\ldots, L_{\max }\right\}$. For each candidate EUP, the OP is evaluated using 599 (17), where the one achieving the minimum OP is selected.

600

\begin{tabular}{lr}
\hline Algorithm 2 1D-Search Algorithm & \\
\hline 1: $L_{d, \text { opt }} \leftarrow 0 ;$ & 601 \\
2: $P_{\text {out,min }} \leftarrow 1 ;$ & 602 \\
3: for $L_{d}=0$ to $L_{\max }$ do & 603 \\
4: $\quad P_{\text {out }}=P_{\text {out }}\left(L_{d}\right) ;$ & 604 \\
5: if $P_{\text {out }}<P_{\text {out,min }}$ then & 605 \\
6: $\quad P_{\text {out }, \text { min }} \leftarrow P_{\text {out }} ;$ & 606 \\
7: $\quad L_{d, \text { opt }} \leftarrow L_{d} ;$ & 607 \\
8: end if & 608 \\
9: end for & 609 \\
\hline
\end{tabular}


610 Specifically, the 1-D EUP-search procedure of Algorithm 2 611 requires $\left(L_{\max }+1\right)$ evaluations of the OP, which is signifi612 cantly lower than that of the 2-D EUP-search of Algorithm 1 613 or the exhaustive search methods. The low complexity of 614 Algorithm 2 accrues from the fact that the EUP functions $615 L_{t}(l)$ investigated may be characterized by a single scalar $L_{d}$, 616 as shown in (16). Therefore, the OP may be expressed as a 617 function of a scalar $L_{d}$, rather than as a vector $\overrightarrow{L_{t}} \triangleq\left\{L_{t}(l) \mid l \in\right.$ $\left.618\left[0, L_{\max }\right]\right\}$. In Section IV-A, we will compare the OP of the 619 proposed 2D-search and 1D-search Algorithms 1 and 2 to a pair 620 of state-of-the-art EUPs found in the literature, namely, to the 621 best-effort policy [6] and to the asymptotic optimal policy [7].

622

623

624 In the previous section, the EUPs conceived for minimizing 625 the OP of P2P networks were investigated. Here, we continue 626 by investigating the EUP design of an SDMA prototype net627 work. Compared to the P2P network, the outage events of 628 different EH-SNs are correlated, but a centralized optimization 629 would impose an excessive complexity. Even if a subopti630 mal 1-D EUP search space is adopted for each EH-SN, an $631 M$-dimensional search space is required for an SDMA network 632 of $M$ EH-SNs, which is generally not practical. In addition, the 633 global knowledge of the channel quality between each EH-SN 634 and the DN, as well as the statistical distribution of the energy 635 arrival rates, should be available at a central controller node, 636 which also imposes a high side-information signaling overhead 637 and complexity. Furthermore, for traditional non-EH SDMA 638 networks, the closed-form OP expressions are not available in 639 the open literature for generalized SDMA networks having $M$ $640 \mathrm{SNs}$, since the derivation of the closed-form OP expressions for 641 SDMA-EH networks is quite challenging.

642 Therefore, we embark on the OP analysis of an SDMA 643 network relying on ML detection and use the minimum-SNR 644 (min-SNR) approximations to arrive at the approximate OP 645 of our SDMA networks, which has been documented in [20] 646 and [21]. It will be shown that the min-SNR approximations 647 are accurate in predicting the OP of the SDMA networks. 648 Given an SDMA network comprised of $M$ EH-SNs and a $649 \mathrm{DN}$, we decompose the approximate joint OP of SDMA into 650 a product of $M$ mutually independent OP components, each of 651 which corresponds to a P2P-EH-network counterpart. Then, we 652 propose a DEUPO protocol, in which each EH-SN is capable of 653 optimizing its own EUP based on the 2-D and 1-D EUP-search 654 algorithms in Section II, using the statistics of its own uplink 655 (UL) channel and its own energy arrival rates, indicating that 656 only local knowledge is required.

\section{A. System Model and OP Formulation}

658 We consider a network of $(M+1)$ nodes, where $M$ SNs $659\left\{S_{m}, 1 \leq m \leq M\right\}$ transmit their individual information to a 660 common DN, and each $\mathrm{SN}$ is equipped with both a harvesting 661 scheme and an EB, as shown in Fig. 1. Again, we assume 662 a narrow-band Rayleigh block-fading channel model, where 663 the fading coefficients remain constant for the duration of a

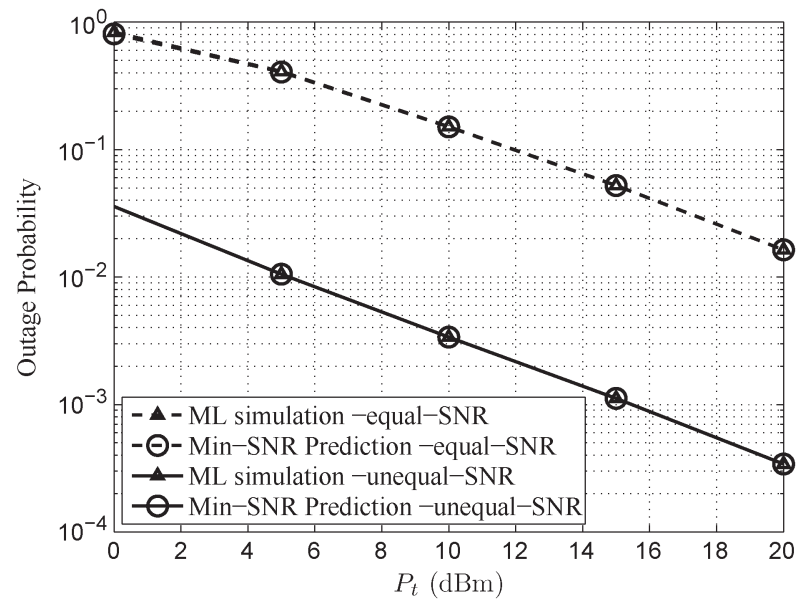

Fig. 3. Accuracy of the OP $\left(P_{\text {out }}\right)$ evaluation using the min-SNR approximations for $M=4, R=0.5 \mathrm{~b} / \mathrm{s} / \mathrm{Hz}$. The distance between the SNs and the DN is $d_{s d}=100 \mathrm{~m}$ and the pathloss exponent is $\beta=3$.

packet and then are faded independently from one packet to 664 another in both time and space. The additive noise imposed by 665 the receivers is modeled by independent zero-mean circularly 666 symmetric complex Gaussian random variables with a variance 667 of unity.

668

The DN is assumed to have perfect channel knowledge and 669 adopts ML detection. All the SNs transmit their messages 670 concurrently at the rate of $R$. The $\mathrm{SN} S_{m}$ encodes a bit 671 sequence into a codeword and transmits it to the DN, where 672 the DN jointly decodes the codewords received from all the 673 SNs. Therefore, the SN-DN hop may be modeled by a multiple- 674 access channel (MAC), and the criterion used for successful 675 decoding is

676

$$
\sum_{m \in S} R \leq \log \left(1+\sum_{m \in S} \gamma_{m d}\right) \forall S \subseteq\left\{S_{m}, 1 \leq m \leq M\right\}
$$

where $\gamma_{m d}$ represents the instantaneous received SNR of the 677 $S_{m}$-DN link. There are $(M !-1)$ inequalities in (21), and even 678 if a single one of the inequalities in (21) is not satisfied, the 679 transmission over the SN-DN hop becomes erroneous. Hence, 680 when the min-SNR of the $M$ channels spanning from the 681 SNs to the reference node (RN), defined as $\gamma_{s d}^{\min }=\min _{m \in S} \gamma_{m d}$, $682 \quad \mathbf{A Q 4}$ is lower than the threshold $\gamma_{\mathrm{th}}^{s d}=2^{R}-1$ to be exceeded for 683 successful decoding, an outage event occurs. Therefore, we 684 aim for modeling the OP of the $M$-user MAC on the SN-RN 685 hop with the aid of the specific SN-RN link having the min- 686 SNR $\gamma_{s d}^{\min }$.

Specifically, in Fig. 3, we compare the OP of the $M$-user 688 MAC channel using ML detection to that of a single link 689 having the min-SNR $\gamma_{s d}^{\min }$ of the $M$-user system. As shown 690 in Fig. 3, the OP of the two systems obtained by simulation 691 perfectly matches for both the equal-SNR and the unequal-SNR 692 scenarios. Specifically, in the equal-SNR situation, the average 693 channel quality of the link spanning from each SN to the DN 694 is identical, while in the unequal-SNR scenario, the average 695 channel quality is different, where the SNRs of the $M=4696$ links are one, two, four, and eight times higher than that in the 697 
698 equal-SNR situation, respectively. It is shown in Fig. 3 that 699 the exact OP of the $M$-user MAC channel and the predicted 700 OP using the P2P channel associated with the min-SNR are 701 identical for both scenarios. Hence, the OP using the min-SNR 702 approximation may be expressed as follows:

$$
\begin{aligned}
P_{\mathrm{out}, S D} & \approx \operatorname{Pr}\left\{\min _{m \in S} \gamma_{m d}<\gamma_{\mathrm{th}}^{s d}\right\}=1-\operatorname{Pr}\left\{\min _{m \in S} \gamma_{m d} \geq \gamma_{\mathrm{th}}^{s d}\right\} \\
& =1-\prod_{m \in S} \operatorname{Pr}\left\{\gamma_{m d}<\gamma_{\mathrm{th}}^{s d}\right\} \triangleq 1-\prod_{m \in S}\left(1-P_{\mathrm{out}, m d}\right) .
\end{aligned}
$$

\section{B. DEUPO Protocol}

704 Having confirmed the accuracy of the min-SNR approxima705 tion, we are now in the position to formulate the OP minimiza706 tion problem for the SDMA-EH network as follows:

$$
\min _{L_{t, 1}(l), L_{t, 2}(l), \ldots, L_{t, m}(l)} P_{\text {out }, S D}\left(L_{t, 1}(l), L_{t, 2}(l), \ldots, L_{t, m}(l)\right)
$$

707 where $L_{t, 1}(l), L_{t, 2}(l), \ldots, L_{t, m}(l)$ corresponds to the discrete 708 EUPs at the SNs. Equivalently, the minimization problem de709 fined in (23) may be expressed by

$$
\max _{L_{t, 1}(l), L_{t, 2}(l), \ldots, L_{t, m}(l)}\left[1-P_{\text {out }, S D}\left(L_{t, 1}(l), L_{t, 2}(l), \ldots, L_{t, m}(l)\right)\right] .
$$

710 Let us now investigate the formulation of $[1-$ $\left.711 P_{\text {out }, S D}\left(L_{t, 1}(l), L_{t, 2}(l), \ldots, L_{t, m}(l)\right)\right]$ in detail. By using 712 the min-SNR approximation of (22), we have

$$
\begin{aligned}
& {\left[1-P_{\text {out }, S D}\left(L_{t, 1}(l), L_{t, 2}(l), \ldots, L_{t, m}(l)\right)\right]} \\
& \approx \prod_{m \in S}\left[1-P_{\text {out }, m d}\left(L_{t, m}(l)\right)\right] .
\end{aligned}
$$

713 In order to maximize the OF of (24), we may maximize each 714 component of $\left[1-P_{\text {out }, m d}\left(L_{t, m}(l)\right)\right]$. Since they are mutually 715 independent or equivalently, we may minimize each compo716 nent's $P_{\text {out } m d}\left(L_{t, m}(l)\right)$. This is beneficial, because the $m$ th 717 component $P_{\text {out }, m d}\left(L_{t, m}(l)\right)$ corresponds to the OP of a P2P$718 \mathrm{EH}$ link spanning from the $m$ th EH-SN to the DN, while it is 719 independent of both the channel quality and the EUPs adopted 720 by other EH-SNs.

721 Therefore, we may design a DEUPO protocol, in which each 722 EH-SN optimizes its own EUP relying on the proposed 1D723 search and 2D-search algorithms proposed for a P2P link in 724 Section II. Specifically, we design the protocol as follows.

- Acquiring the Energy Arrival Rate and Channel Statistics: In practical applications, the system designer may choose appropriate EHI and CSI estimation algorithms, through which the system may detect the changes, generate a trigger, and decide when to activate its EUP optimization. This is a widely used event-triggered protocol [22], [23]. A simpler solution is to periodically invoke the EUP optimization, according to the instantaneous estimated statistics of both the energy arrival rates and the channels. This is, however, beyond the scope of this paper. Instead, we focus our attention on the issue of 735 deciding the EUP, whenever the optimization is activated. 736 In our analysis, we assume that both the estimated en- 737 ergy arrival rate and the channel statistics are perfectly 738 estimated. Hence, each EH-SN has perfect knowledge of 739 the statistics of energy arrival rate, while the DN has the 740 knowledge of the statistics of the UL channels spanning 741 from each EH-SN. In practice, this knowledge is acquired 742 with the aid of pilot-based channel estimation mechanism 743 and/or prediction methods.

744

- Local EUP Optimization Phase: Each SN sends a 745 request-to-send (RTS) packet to the DN. The DN would 746 send $M$ clear-to-send (CTS) packets to the $M$ SNs, where 747 the channel statistics between the $m$ th EH-SN and the DN 748 would be conveyed in each CTS packet, which is assumed 749 to be perfectly recovered at the EH-SNs. Then, each EH- 750 SN may adopt the 2D-search in Section II-C or the 1D- 751 search in Section II-D to find the approximate EUP for 752 our P2P-EH network. As discussed in the context of (25), 753 our design objective is to minimize the approximate OP 754 of the SDMA-EH network considered.

- Data Transmission Phase: Each EH-SN commences its 756 session, by transmitting to the DN, by relying on its 757 locally optimized EUP.

\section{Numerical Results}

A. P2P Networks

760

As detailed in Sections II-C and D, the OP relies on the 762 following system parameters:

- Statistics of the energy arrival rates: include the average 764 energy arrival rate $\bar{P}_{\text {in }}$ and the recharge cycle $T_{E}$. The 765 distribution of the fading energy arrival directly affects its 766 rate, which is assumed to be exponentially distributed, as 767 in [6] and [7], to facilitate our comparisons with the state- 768 of-the-art benchmarkers proposed in these references. $\quad 769$

- Statistics of the wireless information-transfer chan- 770 nels: again, the wireless channel spanning from the SN 771 to the DN is assumed to obey Rayleigh block fading, 772 although our analysis technique can be applied to arbitrary 773 channel models.

- Parameters of the EH-SN: the EB size $B_{\max }$ and the 775 data transmission rate $R$.

Here, the dependence of the OP on the aforementioned sys- 777 tem parameters will be investigated. In the context of the P2P- 778 $\mathrm{EH}$ networks, the distance between the $\mathrm{SN}$ and the DN is set 779 to $d_{s d}=100 \mathrm{~m}$ and the pathloss exponent to $\beta=3$, while the 780 noise power at the receiver is assumed to be $N_{0}=-80 \mathrm{dBm} .781$ The data transmission rate is set to $R=1 \mathrm{~b} / \mathrm{s} / \mathrm{Hz}$. In the figures, 782 the analytical results are represented by the dashed curves, 783 while the simulation results are shown by the symbols. It should 784 be noted that the discrete step sizes $\varepsilon_{P}$ used for quantifying the 785 OP and for searching for the feasible EUP sets are different. 786 For OP evaluations, $\varepsilon_{P}$ is set for ensuring that we have $L_{\max }=787$ 6400 to guarantee a high accuracy of quantifying the OP, while 788 we have $\varepsilon_{P}$ set to $L_{\max }=200$, when searching for the EUP 789 using Algorithms 1 and 2 to control the search complexity. 790 


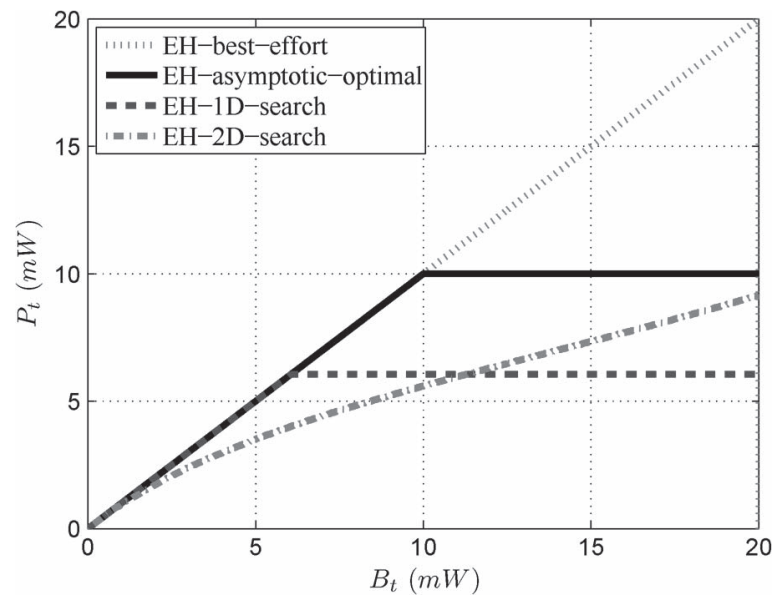

Fig. 4. Transmit power versus the EB state of different EUPs for the P2P network. The average energy arrival rate is $\bar{P}_{\text {in }}=10 \mathrm{dBm}$, and the EB size is $B_{\max }=16 \bar{P}_{\text {in }}$, while we have $R=1 \mathrm{~b} / \mathrm{s} / \mathrm{Hz}$ and $T_{E}=8 T_{C}$.

791 We will demonstrate that the analytical results represented by 792 the dashed curves closely match the simulation results, which 793 indicates that the DMC-based analytical framework is capable 794 of accurately predicting the OP of the P2P-EH networks for all 795 of the EUPs considered.

796 The transmit power versus EB state of the different EUPs 797 are characterized in Fig. 4. It is shown that the best-effort 798 policy proposed in [6] exhibits a slope of 1 , indicating that 799 the currently harvested amount of energy in the EB will be 800 immediately used up for transmission. The $x$-axis $B_{t}$ represents 801 the maximum power that may be supplied, given the amount of 802 energy in the EB for a period of $T_{E}$. The asymptotic optimal 803 policy is based on a combination of two trends: when the 804 amount of energy in the EB satisfies $B_{t}<\bar{P}_{\text {in }}$, the EH-SN 805 transmits by employing the best-effort EUP; otherwise, its EH$806 \mathrm{SN}$ opts for a constant power strategy by choosing a fixed 807 transmit power of $P_{t}=\bar{P}_{\text {in }}$. When the EB size tends to infinity, 808 the asymptotic optimal policy would approach the performance 809 of the constant power policy, indicating that a large EB is 810 capable of converting an EH system into an equivalent classic 811 non-EH system having a constant transmit power of $P_{t}=\bar{P}_{\text {in }}$ 812 [7]. However, when the EB size is finite, the asymptotic optimal 813 policy is no longer optimal in terms of minimizing the OP, as 814 shown in Fig. 5.

815 In Fig. 5, the performance of the EUPs found by the proposed 816 2D-search and 1D-search Algorithms 1 and 2 are compared 817 to that of the best-effort policy and the asymptotic optimal 818 policy proposed in [6] and [7], respectively. It is shown that, 819 for the given configurations, the OP achieved by the proposed 820 algorithms tends to be better than those achieved by the bench821 markers. Specifically, the 2D-search Algorithm 1 performs 822 close to its classic non-EH counterpart, which serves as the 823 lower bound of the OP for the EH systems [7]. At $P_{\text {out }}=0.01$, 824 the EUP found by the 2D-search Algorithm 1 achieves a 3-dB 825 power gain over the asymptotic optimal policy and a 6-dB gain 826 over the best-effort policy. Therefore, if an EH-SN adopts the 827 asymptotic optimal policy, it requires twice the average energy 828 arrival rate harvested from the environment, compared with an 829 EH-SN equipped with the proposed 2D-search algorithm, while

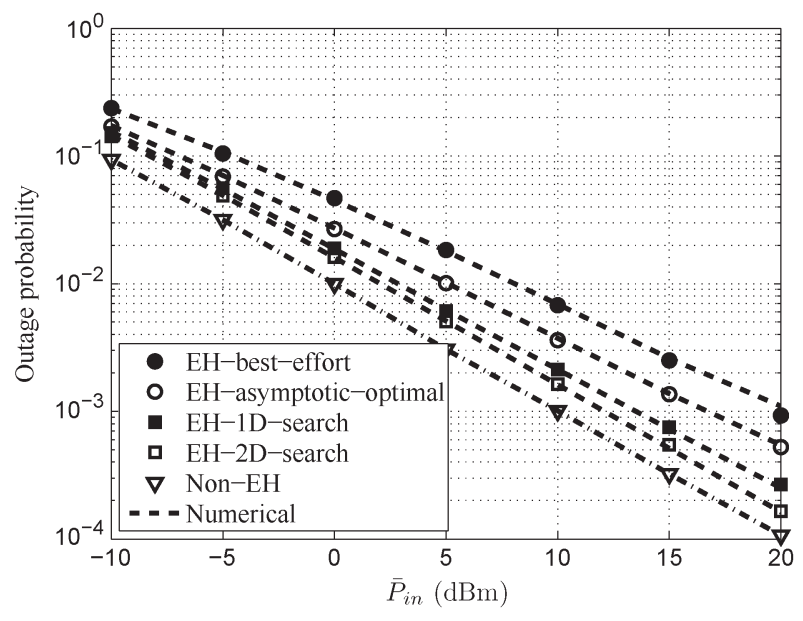

Fig. 5. OP versus average energy arrival rate $\bar{P}_{\text {in }}$ using different EUPs for the $\mathrm{P} 2 \mathrm{P}$ network. The EB size is $B_{\max }=16 \bar{P}_{\text {in }}, R=1 \mathrm{~b} / \mathrm{s} / \mathrm{Hz}$, and $T_{E}=8 T_{C}$. The numerical OP results were evaluated from (11), given the searched EUP.

maintaining the same OP of $P_{\text {out }}=0.01$. This ratio would, 830 in fact, be further increased to four, if the benchmark EH-SN 831 adopts the best-effort policy.

\section{2}

We may conclude that the 2D-search algorithm is capable 833 of most significantly improving the EH-SN's capability to 834 exploit the harvested energy, or to substantially simplify the 835 hardware required for harvesting the energy from the envi- 836 ronment, which is important for applications such as WSNs 837 [1]. For example, the best-effort policy requires a four times 838 higher average energy arrival rate for maintaining an identical 839 outage performance as that using the 2D-search algorithm. 840 Equivalently, the amount of power harvested by the solar panel 841 increases linearly with the area of the solar panel [1], hence 842 requiring a four times larger solar panel. In other words, the 843 2D-search Algorithm 1 allows us to design a sensor node 844 having a solar panel of much smaller size, which has $25 \%$ of 845 the area necessitated by the best-effort policy. Furthermore, as 846 shown in Fig. 5, when the reliability requirements are more 847 stringent, the performance improvements of the proposed EUPs 848 would become more significant in terms of requiring a lower 849 energy arrival rate or a smaller solar panel. Finally, the 1D- 850 search Algorithm 2 is inferior to the 2D-search Algorithm 1, 851 since it exhibits a modest performance degradation of $0.9 \mathrm{~dB} 852$ at $P_{\text {out }}=10^{-2}$. From an alternative perspective, an EH-SN 853 adopting the 1D-search Algorithm 2 may require 1.23 times 854 higher energy arrival rate, which is the price paid for reducing 855 the computational complexity. Therefore, in a WSN application 856 scenario having sensor nodes that have a low computational 857 capability, the 1D-search Algorithm 2 or the simple asymptotic 858 optimal policy may be preferred.

The fundamental reason for the OP improvements of the 860 proposed 2D-search and 1D-search Algorithms 1 and 2 may be 861 inferred from Fig. 6, which represents the PMF of the discrete 862 EB state $l$ for different EUPs. It is observed that all EUPs 863 resulted in near-constant PMF values, apart from the peaks 864 at the states, when the EB was full at $l=L_{\max }$. Compared 865 with the PMF of the best effort and the asymptotic optimal 866 policy, the 2D-search and 1D-search Algorithms 1 and 2 may 867 


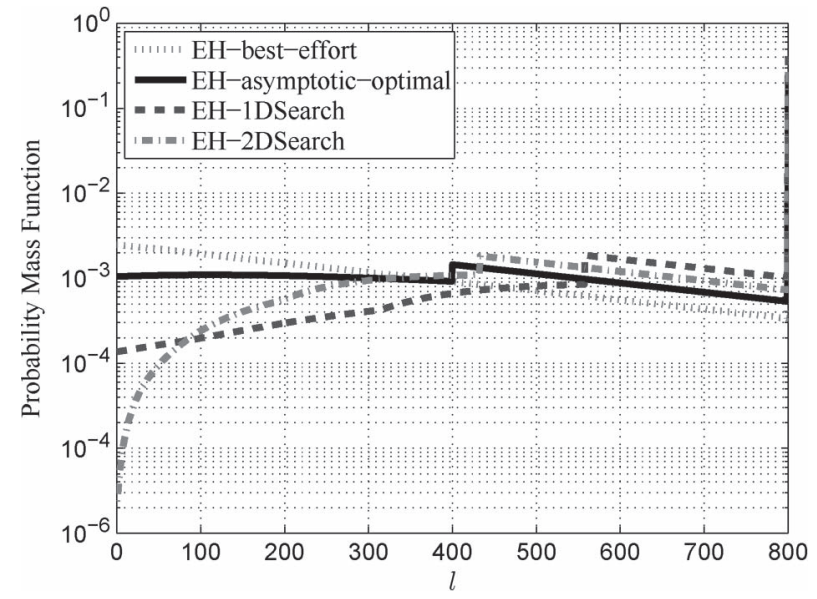

Fig. 6. PMF of the EB states for the P2P network. The average energy arrival rate is $\bar{P}_{\text {in }}=10 \mathrm{dBm}$, while the EB size is $B_{\max }=16 \bar{P}_{\mathrm{in}}, R=1 \mathrm{~b} / \mathrm{s} / \mathrm{Hz}$, and $T_{E}=8 T_{C}$. The results were evaluated via simulations.

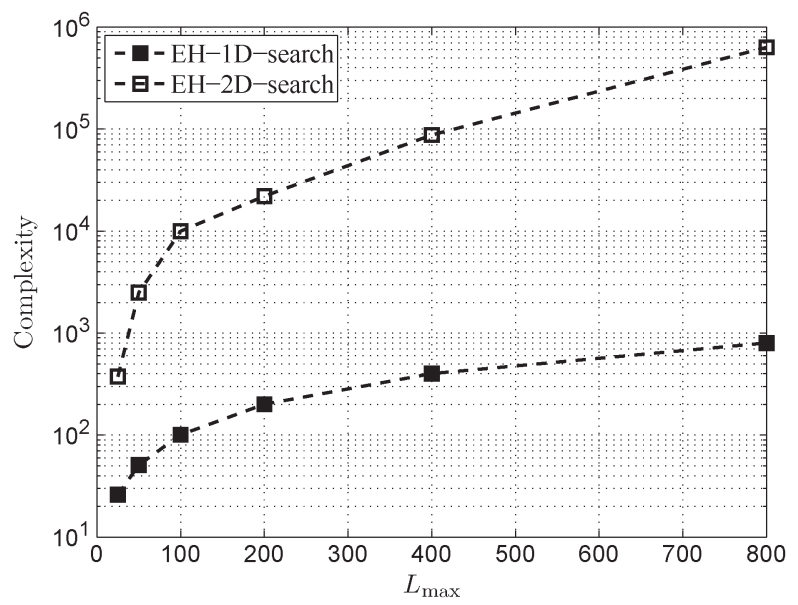

Fig. 7. Computational complexity, in terms of the number of OP evaluations versus the discrete $\mathrm{EB}$ size $L_{\max }$, for the $1 \mathrm{D}$-search and the $2 \mathrm{D}$-search algorithms for the P2P network. The results were evaluated via simulations.

868 be capable of improving the PMF when the EB is small, which 869 reduces the weights $\pi_{l}$ for the relatively large OP components 870 of $P_{e}(l)=\operatorname{Pr}\left\{L_{t}(l)|h|^{2}<L_{\mathrm{th}}\right\}$ in (11), when the discrete 871 transmit power $L_{t}$ is low. Therefore, reshaping the PMF by 872 reducing the contribution of the high OP components and 873 increasing the weights of the low OP components, the overall 874 OP may be beneficially reduced, which is confirmed by the 875 results in Fig. 5. It is also shown that the 1D-search Algorithm 2 876 may be capable of finding an EUP, which performs close to the 877 2D-search Algorithm 1, despite its lower complexity, as shown 878 in Fig. 7.

879 More explicitly, the relationship between the number of OP 880 evaluations and the discrete EB size $L_{\max }$ is illustrated in Fig. 7, 881 for both the 2D-search Algorithm 1 and 1D-search Algorithm 2. 882 Observe that the 1D-search Algorithm 2 drastically reduces the 883 complexity of its 2D-search counterparts. Quantitatively, when 884 the discrete EB size is $L_{\max }=400$, the 1D-search Algorithm 2 885 imposes as little as $0.46 \%$ of the computational complexity 886 compared with that of its 2D-search-based counterpart, while 887 imposing only a modest $0.9-\mathrm{dB}$ loss at $P_{\text {out }}=10^{-2}$, as shown

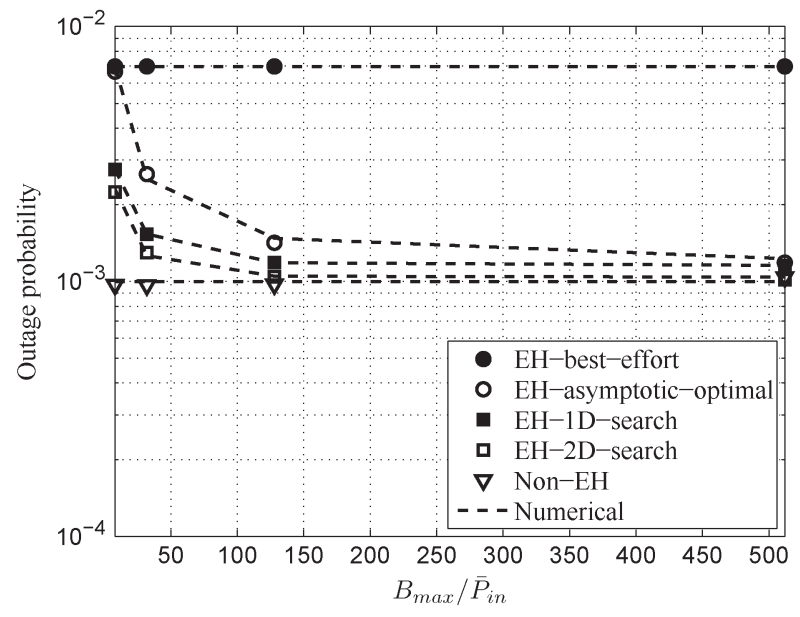

Fig. 8. OP versus the EB size $B_{\max }$ using different energy policies for the $\mathrm{P} 2 \mathrm{P}$ network considered. The average energy arrival rate is $\bar{P}_{\mathrm{in}}=10 \mathrm{dBm}$, $R=1 \mathrm{~b} / \mathrm{s} / \mathrm{Hz}$, and $T_{E}=8 T_{C}$. The numerical OP results were evaluated from (11), given the searched EUP.

in Fig. 5. On the other hand, from an overall energy con- 888 sumption point of view, the computation of the EUP also 889 dissipates a nonnegligible portion of the energy, particularly 890 for users relying on low-end devices. Therefore, the 1D-search 891 Algorithm 2 may be deemed attractive for applications relying 892 on hardware having a low computational capability, such as 893 mobile phones and wireless sensors.

894

In Fig. 8, the impact of EB size $B_{\max }$ is investigated. The 895 horizontal axis is $B_{\max } / \bar{P}_{\text {in }}$. It is shown in Fig. 8 that, when 896 the EB size increases, the OP of both the asymptotic optimal 897 policy proposed in [7] and the EUP relying on our 2D-search 898 Algorithm 1 improves, and they would converge to that of their 899 conventional non-EH counterparts. However, as the EB size 900 $B_{\max }$ increases, the EUP found by the 2D-search Algorithm 1901 may achieve a much better OP, when the EB size is small, 902 and it may converge to that of its classic non-EH counterpart. 903 This confirms the superiority of the proposed search algorithms 904 conceived for EH systems having a finite EB, particularly when 905 the available size of the EB is severely limited.

906

\section{B. Multiple-Access Networks}

907

In Fig. 9, the OP of our SDMA-EH network is investigated, 908 and the EUPs found by the proposed 2D-search and 1D-search 909 algorithms in Section II are compared with those of the best- 910 effort policy and asymptotic optimal policy. It is shown that, for 911 the given configurations, the OPs achieved by the proposed al- 912 gorithms are better than those of the benchmarks. Furthermore, 913 it is shown that the analytical results represented by dashed 914 curves closely match the simulation results, which indicates 915 that the proposed min-SNR approximation and the DMC-based 916 analytical framework are accurate.

Specifically, the 2D-search algorithm performs within $2 \mathrm{~dB} 918$ from its classic non-EH counterpart at $P_{\text {out }}=10^{-2}$, which 919 serves as the lower bound of the OP for EH systems [7]. 920 At $P_{\text {out }}=10^{-2}$, the EUP found by the 2D-search algorithm 921 achieves a 4.6-dB power gain compared with the asymptotic 922 optimal policy and an 8-dB power gain compared with the 923 


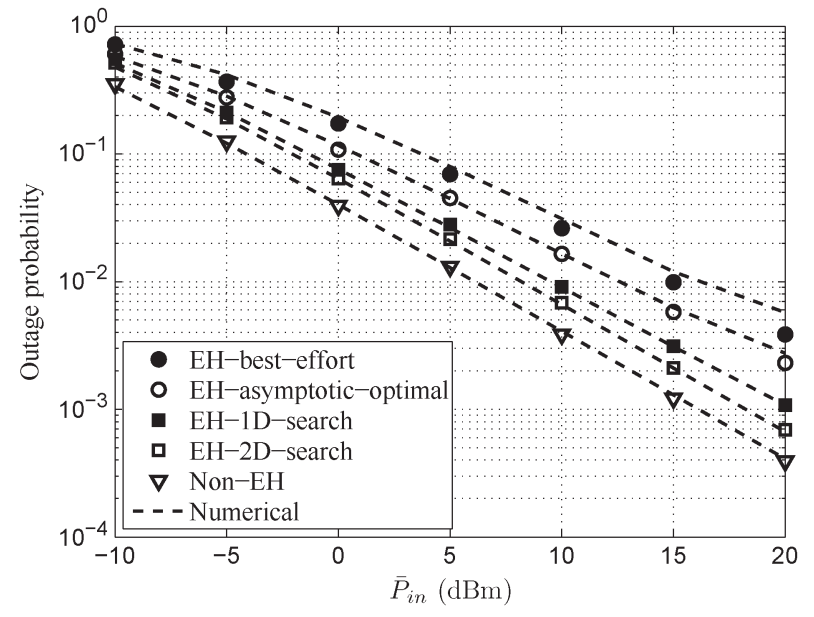

Fig. 9. OP versus average energy arrival rate $\bar{P}_{\text {in }}$ using different energy policies for the SDMA network associated with $M=4$ SNs. The EB size $B_{\max }=16 \bar{P}_{\text {in }}, R=1 \mathrm{~b} / \mathrm{s} / \mathrm{Hz}$, and $T_{E}=8 T_{C}$. The numerical OP results were evaluated from (22), given the searched EUP.

924 best-effort policy. Therefore, if an EH-SN adopts the asymp925 totic optimal EUP, it requires $10^{4.6 / 10} \approx 2.9$ times higher av926 erage energy arrival rates harvested from the environment, as 927 compared to an EH-SN equipped with the proposed 2D-search 928 algorithm at $P_{\text {out }}=10^{-2}$. This ratio would be further increased 929 to a factor of 6.3 , if the benchmark EH-SN adopts the best930 effort policy. The 1D-search algorithm is suboptimal; hence, it 931 exhibits a performance degradation of $1.4 \mathrm{~dB}$ compared to that 932 of the 2D-search algorithm at $P_{\text {out }}=10^{-3}$. From a different 933 perspective, an EH-SN adopting our 1D-search algorithm may 934 require a 1.4 times higher energy arrival rate, which is the price 935 paid for its reduced computational complexity. In our future 936 work, we will jointly consider the optimization of the energy 937 arrival rate and of the power savings of the reduced-complexity 938 algorithms. This might, in fact, favor the 1-D algorithm over its 939 2-D counterpart.

940 Finally, we investigate the effects of the number of EH-SNs 941 on the OP in SDMA-EH networks. It is shown in Fig. 10 that, as 942 the number of SNs $M$ increases, the OP of all EUPs is reduced. 943 However, the EUPs found by the proposed 2D-search and 944 1D-search algorithms always outperform both the asymptotic 945 optimal policy and the best-effort policy. The results allow 946 the SDMA-EH network to accommodate more users, while 947 maintaining the same reliability. For example, if a maximum 948 OP of $P_{\text {out }}=10^{-2}$ is tolerable in the SDMA-EH network, 949 both the best-effort policy and the asymptotic policy may be 950 capable of supporting $K=2$ and 4 users, while the 1D-search 951 and the 2D-search algorithms support more than $K=8$ users 952 simultaneously. To conclude, given the proposed 1D-search and 953 2D-search algorithms, our receiver is capable of simultaneously 954 offering reliable services for significantly more EH users.

\section{CONCLUSION}

956 In this paper, we have summarized the state-of-the-art EUP 957 design aiming for minimizing the OP of P2P-EH networks 958 reported in the literature, and then, we have proposed two 959 novel algorithms, which are capable of exploiting the harvested

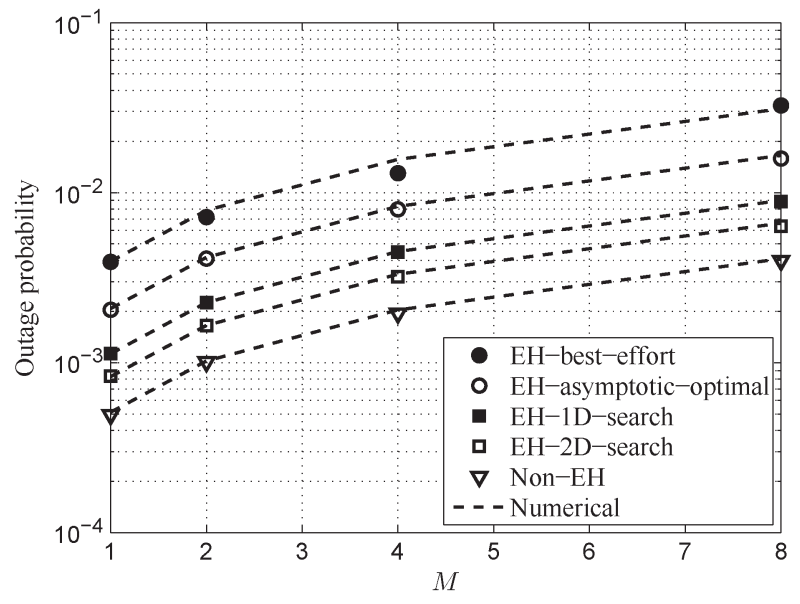

Fig. 10. OP versus average energy arrival rate $\bar{P}_{\text {in }}$ using different energy policies for the SDMA network associated with different number of SNs $M=1,2,4,8$. The average energy arrival rate is $\bar{P}_{\text {in }}=10 \mathrm{dBm}$, the EB size is $B_{\max }=16 \bar{P}_{\text {in }}, R=1 \mathrm{~b} / \mathrm{s} / \mathrm{Hz}$, and $T_{E}=8 T_{C}$. The numerical OP results were evaluated from (22), given the searched EUP.

energy stored in a finite EB, where we showed that, using the 960 proposed algorithms, the achievable OP outperforms the state- 961 of-the-art benchmark systems found in the literature. Further- 962 more, upon invoking the proposed min-SNR approximation, the 963 algorithms advocated were invoked for SDMA-EH networks, 964 where we designed a DEUPO protocol. With the advent of the 965 DEUPO protocols proposed in this paper, our proposed 1D- and 966 2D-search algorithms require a significantly reduced energy 967 arrival rate at a given target $\mathrm{OP}$. 968

\section{REFERENCES}

969

[1] S. Sudevalayam and P. Kulkarni, "Energy harvesting sensor nodes: Sur- 970 vey and implications," IEEE Commun. Surveys Tuts., vol. 13, no. 3, 971 pp. 443-461, 3rd Quart. 2011.

972

[2] O. Ozel, K. Tutuncuoglu, J. Yang, S. Ulukus, and A. Yener, "Transmis- 973 sion with energy harvesting nodes in fading wireless channels: Optimal 974 policies," IEEE J. Sel. Areas Commun., vol. 29, no. 8, pp. 1732-1743, 975 Sep. 2011.

976

[3] J. Yang and S. Ulukus, "Optimal packet scheduling in an energy har- 977 vesting communication system," IEEE Trans. Commun., vol. 60, no. 8, 978 pp. 220-230, Jan. 2012.

979

[4] T. Zhang et al., "A cross-layer perspective on energy harvesting aided 980 green communications over fading channels," IEEE Trans. Veh. Technol., 981 to be published.

[5] C. Huang, R. Zhang, and S. Cui, "Outage minimization in fading channels 983 under energy harvesting constraints," in Proc. IEEE ICC, Jun. 2012, 984 pp. 5788-5793.

985

[6] S. Luo, R. Zhang, and T. J. Lim, "Optimal save-then-transmit protocol for 986 energy harvesting wireless transmitters," IEEE Trans. Wireless Commun., 987 vol. 12, no. 3, pp. 1196-1207, Mar. 2013.

988

[7] N. Zlatanov, R. Schober, and Z. Hadzi-Velkov, "Asymptotically optimal 989 power allocation for energy harvesting communication networks," IEEE 990 Trans. Inf. Theory, submitted for publication.

[8] C. Huang, R. Zhang, and S. Cui, "Optimal power allocation for outage 992 probability minimization in fading channels with energy harvesting con- 993 straints," IEEE Trans. Wireless Commun., vol. 13, no. 2, pp. 1074-1087, 994 Feb. 2014.

[9] Y. Mao, G. Yu, and Z. Zhang, "On the optimal transmission policy in 996 hybrid energy supply wireless communication systems," IEEE Trans. 997 Wireless Commun., vol. 13, no. 11, pp. 6422-6430, Nov. 2014.

[10] P. He, L. Zhao, S. Zhou, and Z. Niu, "Recursive waterfilling for wireless 999 links with energy harvesting transmitters," IEEE Trans. Veh. Technol., 1000 vol. 63, no. 3, pp. 1232-1241, Mar. 2014.

1001

[11] C. K. Ho and R. Zhang, "Optimal energy allocation for wireless communi- 1002 cations with energy harvesting constraints," IEEE Trans. Signal Process., 1003 vol. 60, no. 9, pp. 4808-4818, Sep. 2012. 
1005 [12] J. Yang and S. Ulukus, "Optimal packet scheduling in a multiple access 1006 channel with energy harvesting transmitters," J. Commun. Netw., vol. 14, 1007 no. 2, pp. 140-150, Apr. 2012.

1008 [13] F. Iannello, O. Simeone, and U. Spagnolini, "Medium access control 1009 protocols for wireless sensor networks with energy harvesting," IEEE 1010 Trans. Commun., vol. 60, no. 5, pp. 1381-1389, May 2012.

1011 [14] H. Li, C. Huang, S. Cui, and J. Zhang, "Distributed opportunistic schedul1012 ing for wireless networks powered by renewable energy sources," in Proc. 1013 IEEE Infocom, 2014, pp. 1-9.

AQ7 1014 [15] Z. Wang, V. Aggarwal, and X. Wang, "Iterative dynamic water-filling 1015 for fading multiple-access channels with energy harvesting," pp. 1-34, 1016 Jan. 2014.

1017 [16] D. Tse and P. Viswanath, Fundamentals of Wireless Communication. 1018 Cambridge, U.K.: Cambridge Univ. Press, 2005.

1019 [17] R. Gallager, "Discrete Stochastic Processes," MIT OpenCourseWare: 1020 Massachusetts Inst. Technol., Cambridge, MA, USA, 2011

1021 [18] I. Krikidis, S. Timotheou, and S. Sasaki, "RF energy transfer for coopera1022 tive networks: Data relaying or energy harvesting?," IEEE Commun. Lett., 1023 vol. 16, no. 11, pp. 1772-1775, Nov. 2012.

1024 [19] V. Kulkarni, A. Forster, and G. Venayagamoorthy, "Computational intel1025 ligence in wireless sensor networks: A survey," IEEE Commun. Surveys 1026 Tuts., vol. 13, no. 99, pp. 1-29, 1st Quart. 2011.

1027 [20] B. Zhang, J. Hu, Y. Huang, M. El-Hajjar, and L. Hanzo, "Outage analysis 1028 of superposition modulation aided network coded cooperation in the pres1029 ence of network coding noise," IEEE Trans. Veh. Technol., vol. 64, no. 2, 1030 pp. 493-501, Feb. 2014.

AQ8 1031 [21] B. Zhang, C. Dong, J. Lei, M. El-hajjar, L.-l. Yang, and L. Hanzo, "Buffer1032 aided relaying for the multi-user uplink: Outage analysis and power allo1033 cation," IEEE Trans. Veh. Technol., submitted for publication.

1034 [22] C. Intanagonwiwat, R. Govindan, D. Estrin, J. Heidemann, and F. Silva, 1035 "Directed diffusion for wireless sensor networking," IEEE/ACM Trans. 1036 Netw., vol. 11, no. 1, pp. 2-16, Feb. 2003.

1037 [23] Y. Hou, H. Sherali, and S. Midkiff, "On energy provisioning and relay 1038 node placement for wireless sensor networks," IEEE Trans. Wireless 1039 Commun., vol. 4, no. 5, pp. 2579-2590, Sep. 2005.

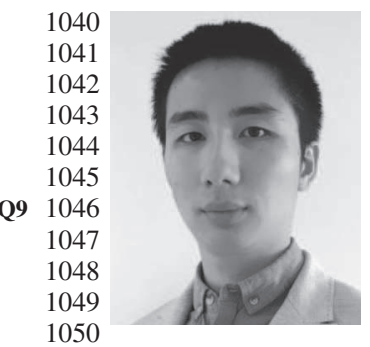

Bo Zhang received the B.Eng. degree in information engineering from the National University of Defense Technology, Changsha, China, in 2010 and the Ph.D. degree in wireless communications from the University of Southampton, Southampton, U.K., in 2015.

$\mathrm{He}$ is currently with the National University of Defense Technology. His research interests in wireless communications include the design and analysis of cooperative communications, multipleinput-multiple-output systems, and network-coded systems.

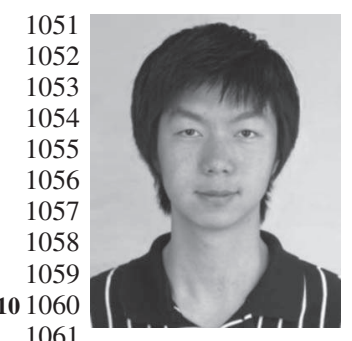

Chen Dong received the B.S. degree in electronic information sciences and technology from the University of Science and Technology of China, Hefei, China in 2004; the M.Eng. degree in pattern recognition and automatic equipment from the University of Chinese Academy of Sciences, Beijing, China, in 2007; and the Ph.D. degree from the University of Southampton, Southampton, U.K., in 2014.

$\mathrm{He}$ is a Postdoctoral Researcher with the University of Southampton His research interests include applied mathematics, relay systems, channel model1062 ing, and cross-layer optimization.

1063 Dr. Dong received a scholarship under the U.K.-China Scholarships for 1064 Excellence Program, and he was awarded the Best Paper Award at the Fall 10652014 IEEE Vehicular Technology Conference.

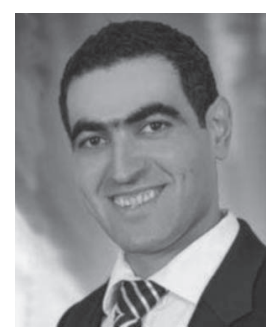

Mohammed El-Hajjar received the B.Eng. degree 1066 in electrical engineering from the American Uni- 1067 versity of Beirut, Beirut, Lebanon, in 2004 and the 1068 M.Sc. degree in radio frequency communication sys- 1069 tems and the Ph.D. degree in wireless communi- 1070 cations, both from the University of Southampton, 1071 Southampton, U.K., in 2005 and 2008, respectively. 1072

After his Ph.D. studies, he joined Imagination 1073 Technologies as a Design Engineer, where he worked 1074 on designing and developing Imagination's multi- 1075 standard communications platform, which resulted 1076 in three patents. In January 2012, he joined the School of Electronics and 1077 Computer Science, University of Southampton, where he is a Lecturer in 1078 the Southampton Wireless Research Group. He has published a Wiley-IEEE 1079 book and more than 50 journal and international conference papers. His 1080 research interests are mainly on the development of intelligent communications 1081 systems, including energy-efficient transceiver design, cross-layer optimization 1082 for large-scale networks, multiple-input-multiple-output systems, millimeter- 1083 wave communications, and radio-over-fiber systems.

1084

Dr. El-Hajjar has received several academic awards, including the Dean's 1085 Award for Creative Achievement, the Dorothy Hodgkin Postgraduate Award, 1086 and the 2010 IEEE International Conference on Communications Best Paper 1087 Award.

1088

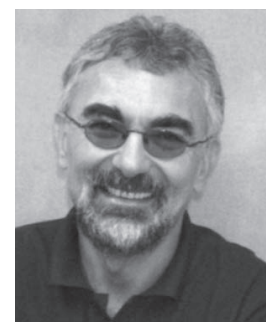

Lajos Hanzo (M'91-SM'92-F'04) received the 1089 Master's degree in electronics, the Ph.D. degree, and 1090 the Doctor Honoris Causa degree from the Technical 1091 University of Budapest, Budapest, Hungary, in 1976, 1092 1983, and 2009, respectively, and the D.Sc. degree 1093 from the University of Southampton, Southampton, 1094 U.K., in 2004.

1095

During his career in telecommunications, he 1096 has held various research and academic posts in 1097 Hungary, Germany, and the U.K. Since 1986, he has 1098 been with the School of Electronics and Computer 1099 Science, University of Southampton, Southampton, U.K., where he holds the 1100 Chair in telecommunications. He was a Chaired Professor with Tsinghua 1101 University, Beijing, China. He is the coauthor of 20 John Wiley/IEEE Press 1102 books on mobile radio communications, totalling in excess of 10000 pages, and 1103 has published more than 1400 research entries on IEEE Xplore. He is currently 1104 directing an academic research team, working on a range of research projects 1105 in the field of wireless multimedia communications sponsored by industry, 1106 the Engineering and Physical Sciences Research Council (EPSRC) U.K., the 1107 European IST Program, and the Mobile Virtual Centre of Excellence, U.K. He 1108 is an enthusiastic supporter of industrial and academic liaison, and he offers a 1109 wide range of industrial courses.

Dr. Hanzo has acted as a Technical Program Committee Chair for IEEE con- 1111 ferences, presented keynote lectures, and has received a number of distinctions. 1112 $\mathrm{He}$ is the Governor of the IEEE Vehicular Technology Society and the Past 1113 Editor-in-Chief of the IEEE Press. 


\section{AUTHOR QUERIES}

\section{AUTHOR PLEASE ANSWER ALL QUERIES}

$\mathrm{AQ1}=$ Please check if the expanded form of RC-UK is properly captured.

AQ2 = Please check if "EH-P2P" is properly captured as "P2P-EH" to maintain consistency in the text.

AQ3 = Please check if "block Rayleigh fading channel" is properly changed to "Rayleigh block-fading channel" to maintain consistency in the text.

AQ4 = Please check if the expanded form of RN is properly captured.

AQ5 = Please provide publication update in ref. [4].

AQ6 = Please provide publication update in ref. [7].

AQ7 = Please provide title of the publication in Ref. [15].

AQ8 = Please provide publication update in ref. [21].

AQ9 = Please check if this sentence is properly inserted to maintain consistency with the current affiliation; otherwise, kindly provide changes.

AQ10 $=$ Please check if the expanded form of FREng, FIET, and EURASIP and VTS and TPC are properly captured.

END OF ALL QUERIES 